\title{
Initiation mathématique à l'hydrologie fluviale
}

\section{Mathematical initiation to river hydraulics}

\author{
PAR A. GOUTAGNE (1)
}

\author{
«Et moi, dil le Pelit Prince, si \\ jarais einquante-trois minutes à per- \\ dre, je marcherais vers une fontaine.
}

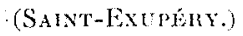

\begin{abstract}
Mathématiques et hydrologie. - Distributions statistiques: rappel des definitions, etude of comparison d'un certain nombre de courbes de distributions theriques. - Principes et ressources de l'étude des corrélations et des covariations.
\end{abstract}

\begin{abstract}
Mathematics and Iydrology. - Statistical distributions : enumerations of definitions, study and comparison of some theoretical distribution curves. Theory and resources of investigations on correlation and conariation.
\end{abstract}

\section{I. - CONSIDÉRATIONS GÉNÉRALES}

Au début de celle causerie sur l'application des mathématiques aux investigations d'hydrologie fluviale, je me permettrai de citer une page de votre éminent professeur et de mon excellent ami, M. Pardé (Fleuves et Rivières, 1933) :

«Est-il besoin de souligner la portée pratique de l'hydrologie fluviale, en un siècle où l'on utilise de plus en plus les eaux courantes pour des fins variées : irrigation, navigation et surtout production d'énergie? Il va de soi que les aménagements des rivières sont conditionnés par les régimes.

"L’intérêt scientifique de l'hydrologie fluviale n'est pas moins clair. Pour bien comprendre un régime, il faut ouvrir sur lui plusieurs fenètres. Les débits, base de l'Hydrologie, sont mesurés et calculés à l'aide de l'hydranlique, e'est-à-dire finalement, au moyen de la physique et des mathématiques. Ils s'expliquent par la morphologie, ou étude du relief terrestre, par la météorologie, par la géologie, par la couverture végétale, done par la botanique.
«En somme, si la science hydrologique possède son originalité, elle dépend d'autres disciplines, dont la plupart se rapportent à la géographie. Elle figure avant tout parmi les maîlres rameaux de la géographie physique, el autant que les branches voisines, elle importe à la géographie humaine, puisque l'homme utilise les cours d'eau ou les redoute.

"D'autre part, le régime des fleuves el rivières donne une captivante impression de vie. Un cours d'eau, comme un climat, est doué souvent d'un caractère complexe comme celui des êtres humains. Le plus ignorant peut constater certaines manifestations lentes ou rapides de cette persomnalité. Les épisodes de l'hydrologie ressemblent aux phases d'un roman ou d'un drame.

«Enfin, le désordre apparent de ces phénomènes, comme les éléments contradictoires d'une psychologie, est soumis a des lois rigoureuses, et pour la plupart connues dès maintenant. Aussi, malgré le caractère seulement approximatif de beaucoup de chiffres hydrologiques, les conclusions que l'on peut fonder sur eax comptent déjà parmi les plus précises de celles dont la physi- 
On ne peut mieux délinir la complexité et la multiplicité des aspects de l'hydrologie fluviale, dont l'étude nécessite l'intervention de disciplines scientifiques les plus diverses. Parmi ces disciplines, les synthétisant, les amalgamant en quelque sorte, les dominant mème, - - les mathématiques sont le langage de toutes les seicnces, et une discipline ne mérite vraiment le nom de seience qu'à partir du jour où le nombre $\mathrm{y}$ a pénétré, a écrit P. Montel -- parmi ces disciplines, dis-je, il en est une sur laquelle je me propose aujourd'hui d'insister plus particulièrement, j'ai nommé la discipline mathématique qui permet, précisément, grâce aux notions fondamentales de probabilité et de corrélation, qui sont à la base de toute la physique moderne, et ont pénétré mème dans le domaine des seiences biologiques, morales, sociales, économiques, de dégager du désordre apparent des phénomènes naturels et autres, dont parle fort justement M. Paroé, des lois qui, certes, sont loin d'être aussi rigoureuses qu'il le dit, mais qui aident à la compréhension du monde et de ses phénomènes de toute nature.

Ces lois, effectivement, ne sont que des lois moyennes, c'est-à-dire des lois exprimant le comportement moyen d'objets dont nous ne pouvons définir et prévoir le comportement individuel; des lois qu'on ne peut qualifier de fonctionnelles, comme celles de la mécanique céleste, mais statistiques, c'est-à-dire empiriques, aléatoires, concrétisées par des graphiques ou des formules qui contiennent les unes et les autres, à un degré plus ou moins grand, cette variable un peu déconcertante, énigmatique, « la probabilité » qui représente, en quelque sorte, la synthèse de toutes les causes enchevêtrées dans la nature, dont nous ne pouvons discriminer et différencier les effets et qu'en langage vulgaire on appelle «le hasard».

Le calcul des probabilités offre une contradiction dans les termes mêmes qui servent à le définir, comme l'a fait remarquer $H$. Porncarí qui a ajouté : «Si je ne craignais de rappeler ici un mot souvent répété, je dirais qu'il nous enseigne surtout une chose, e'est que nous ne savons rien ». Mais tel qu'il est, malgré les obscurités qui subsistent dans cette notion fondamentale, malgré les controverses auxquelles elle donne lieu entre «objectivistes » et « subjectivistes », la fécondité et la validité de ses applications ne peuvent être ni ébranlées, ni suspectées et par elle les mathématiques sont de plus en plus mêlées à la vie des individus, des collectivités, des peuples.

Remarquons, d'ailleurs, que la notion de probabilité a pris sa source dans la méditation des phénomènes concrets, aussi bien ceux de la vie courante que ceux des sciences physiques, bio- logiques, économiques, psychologiques, sociales, contrairement à beaucoup d'autres notions mathématiques qui ont pris naissance dans le monde des abstractions.

Illustrons, si vous le voulez bien, ces quelques considérations générales par quelques citations, dont nous nous sommes d'ailleurs fortement inspiré pour les formuler :

\section{De P. MONTEL :}

«Les mathématiques se sont mèlées à la vie des individus et des peuples par la notion de probabilité. Nombre de nos décisions personnelles, nombre de mesures collectives dérivent de cette notion. La marche d'organismes comme les Compagnies d'Assurances et les banques, d'appareils comme le téléphone automatique, est soumise à ses lois. La notion de probabilité s'introduit même au cour mème de certaines parties de la physique moderne. Elle exprime des lois déduites des valeurs moyennes des actions d'une multitude de molécules dont on ignore les conditions initiales et les mouvements.

«Il y a une science du hasard. Pour l'édifier, il a fallu préciser ce terme un peu confus de probable, de façon à l'introduire dans les calculs, et étudier, comme l'a fait Pascal, les jeux les plus simples, celui de pile ou face, avec une pièce de monnaie, ou de rouge ou noir, à la roulette. La probabilité d'un événement est le rapport du nombre de cas favorables au nombre de cas possibles : il varie entre la valeur 1 qui correspond à la certitude, et la valeur 0 qui correspond à l'impossibilité. Lorsque la probabilité est très petite, nous sommes enclins à la négliger. Le risque d'être écrasé dans la rue d'une grande ville est de quelques millionièmes. Si on ne le négligeait pas, on ne sortirait plus de chez soi. Le risque de mort par un accident de chemin de fer est, en France, d'un millionième environ, pour le voyage de Paris-Nice. Qui s'en soucie? Cependant, la chance de gagner le gros lot à la Loterie Nationale est inférieure à un millionième et chaque détenteur de billet y pense avec complaisance, car notre esprit se tourne vers les circonstances heureuses avec plus de faveur que vers les événements funestes.

"Nous sommes si fermement attachés à la notion de probabilité que nous nous attendons à voir les phénomènes la traduire fidèlement. Après une longue série de rouges, nous attendons le retour de la noire. Cette exigence de notre esprit a aussi sa loi, celle des grands nombres à laquelle obéissent les écarts. Ces écarts peuvent être d'autant plus grands, les séries d'autant plus longues que le nombre des parties est plus élevé. C'est ce qui explique la ruine du joneur et l'échec de toute martingale. 
"Par la statistique el la probabilité, les mathématiques agissent sur la vie économique et sociale, sur l'éducation et dans cette psychologie de la technique actuellement en faveur. Elles regissent aussi les associations biologiques animales et végétales, et il existe une mathématique de la lutte pour la vie. Cette thérie a donné une vigueur nouvelle à la doctrine de la sćlection naturelle, et les problèmes relatifs à l'évolution ont progressé dans ces dernieres années plus qu'ils ne l'avaient fait durant le demi-siecle précédent.

« Je viens de parler du ròle joué par la probabilité dans la vie des individus et des peuples. L'Etat et les collectivités essaient de compenser, au moyen d'assurances, les risques de mort, d'aceident, de maladie ou de vol. En offrant ainsi moins d'incertitude à la condition humaine, on court le risque d'atíablir le goût du risque qui a conduit l'homme aux grandes découvertes et aux puissantes aventures; et de retarder le progrès. 》

\section{De P. Monter également :}

«Si les mathématiques sont partout, si chacun de nous les emploie chaque jour, quelle est donc la tâche des mathématiciens?

«Ils font autre chose que des calculs. Ils éludient de plus en plus profondément les lois qui régissent les rapports entre les nombres, et des problèmes nouveaux se lèvent sur leurs pas à mesure qu'ils en résolvent.

"Leurs découvertes demeurent parfois inutilisées durant des siècles, outils qui attendent lat main de l'ouvrier. Leur science s'accroit par juxtaposition des derniers résultats aux précédents, alors que les sciences de la nature et les sciences humaines se développent en édifiant des théries nouvelles sur les ruines des anciennes. Dans la cité mathématique, on perce des avenues nouvelles en conservant les vieilles constructions qu'il s'agit d'aménager. Cotte cité est partagée en quelques quartiers; celui des nombres et des grandeurs que ces nombres mesurent, celui des lois et des fonctions qui les expriment, celui du dóterminisme et des équations différentielles, permettant de construire l'être mathématique tout entier, à partir d'une de ses cellules, celui des espaces et de leur géométrie, celui du hasard ol des probabilités.

"La joie esthétique qu'apporte aux mathématiciens la contemplation de leur cité est leur véritable récompense. La solidité de leurs constructions défie le temps. Devant le renouvellement incessant des doctrines et des écoles qui régissent les sciences de la nature et celles de l'homme, seules demeurent immobiles les formes de la mathématique et les formes de l'Art.
« Les mathématiques sont le langage de toutes les sciences, el une discipline ne mérite vraiment le nom de science qu'à partir du jour où le nombre y a pénétré. Elles nous donnent l'exemple te plus étendu de cette interdépendance des sciences que l'Association Française pour l'Avancement des Sciences a pour but principal de développer. »

\section{De F. de Lionnais :}

« Le calcul des probabilités prit naissance au xvi" siècle, comme la séométrie analytique, l'analyse combinatoire et le calcul infinitésimal. Le xvir siècle en ćlimina les principales embùches et lui donna une forme théorique, mais ne fit qu'entrevoir ses applications. Au xrx" siècle, il se révéla un instrument irremplaçable dans l'étude de certains phénomenes physiques, comme la cinćtique des gaz. La physique du $\mathrm{xx}^{*}$ siècle a donné son libre essor à cetle surprenante théoric qui semble avoir été crééc uniquement à son usage. En ruinant les formes, devenues trop étriquées, du déterminisme classique, et en les remplaçant par des formes plus souples, elle s'est adressée au calcul des probabilités pour assurer aux lois fondamentales de la nouvelle mécanique les seules fondations qu'elle puisse désormais posséder. 》

\section{De EDDington :}

"La physique n'est plus maintenant attachée a un cadre de lois impliquant le déterminisme. »

\section{De LANGERON :}

«Il n’y a non pas crise de déterminisme, mais crise du mécanisme, auquel nous avons attribué une trop grande généralité.»

\section{De Max Pranck :}

* Un événement est conditionné causalement, quand il peut être prédit arec certitude.

"La valeur douteuse des prédictions météorologiques est passée en proverbe, et cependant aucun météorologiste ne se résoudra à renoneer à considérer les phénomines atmosphériques comme déterminés causalement.

«On est porté à considérer la recherche de la probabilité comme la tâche qui incombe avant tout au physicien. La notion de probabilité acquiert ainsi une importance primordiale. Elle devient le fondement de toute la physique.»

\section{Du Comte dr Gasparin :}

"La prévoyance des faits futurs n'est que le résultat d'un calcul de probabilités. » 


\section{De J. Chevalier :}

"Naguèc, la mécanique céleste postulait un déterminisme tel que la comnaissance de létat actuel du monde suffisail à déterminer son èvolution ultérieure, la position el la vitesse de chacun de ses atomes dans l'inlini du temps. Mais cela n'est vrai que dans la mécanique céleste, qui réalise un ordre idéal, quasi soustrait au temps. Le déterminisme des phénomènes physiques n'est vrai qu'en gros, c'est dire qu'il revèt une forme statistique. 'Tont est fait par des individus, tout se passe par discontinuité et nous ne pouvons rien connaître que par moyennes, nous ne pouvons appréhender que des ensembles. Nos lois n'expriment que le comportement moyen. d'objets dont nous ne pouvons définir le comportement individael; notre expérience el notre science qui se fonde sur elle sont impuissantes à franchir le seuil du domaine où la nature agit en nous dérobant le principe de ses ouvres ot le secret de sa spontanéité créatrice... Il y a juste assez de déterminisme dans le monde pour permettre a notre action son efficacité; il n'y en a pas trop pour la contraindre.

"La nature ne nous présente pas de lois causales, mais de simples lois de probabilité : c'est que le déterminisme absolu, à supposer qu'il existe, est absolument inaccessible.»

\section{De L. DE BRogle: :}

« La physique ne peut pas prédire les événements futurs avec exactitude, mais seulement los événements possibles et leurs probabilités relatives.

«On ne saurait affirmer aujourd'hui qu'il n'y a pas un déterminisme sous-jacent, mais tous les efiorts faits pour le dégager ayant échoué, rien ne nous impose l'idée que ce déterminisme existe réellement; la plupart des physiciens pensent que l'indétermination récemment mise en évidence est essentielle. Pour eux, chaque fois que se produit un phénomène observable, il y aurait une sorte de choix entre diverses possibilités et la seule chose qui obéirait à des lois rigoureuses, ce serait la probabilité des divers choix possibles.»

Pour en finir avec ces généralités sur l'importance du calcul des probabilités, précisons un point qui a donné lieu à certaines objections, inspirées d'ailleurs par le meilleur bon sens.

On reproche a certaines formules théoriques, par lesquclles les statisticiens extrapolent, andela des limites de l'observation, certaines données expérimentales, d'impliquer la possibilité de phénomènes d'une intensité incompatible avec les contingences naturelles les plus évidentes. Nous rappelons la boutade de l'ingénieur américain HorTox, souvent citée : « A small stream can not produce a major Mississipi River flood, for much the same reason that an ordinary baimyard fowl cannot lay an egg a yard in diameter. It would transcent nature's capabilities under the circonstances.

Cette objection est plus dogmatique qu'effective. En langage mathématique, dire d'un phénoméne que sa probabilité est très petite (d'une petitesse qui, dans chaque cas, doit ètre précisée), c'est dire qu'il est ou doit ètre constdéré comme impossible, en vertu de la loi que M. BoREL a appelée "la loi unique du hasard».

Voici d'ailleurs comment s'exprime M. Robert Fortet, professeur à la Faculté des Sciences de Caen, dans un ouvrage récent : « Pour que l'application du calcul des probabilités à un phénomine concret soit possible, et contròlable par l'expérience, il suffit d'accepter une loi unique, que nous appellerons, abec M. BOREs, la « loi unique du hasard $\gg$, el qu'on peut énoncer ainsi : "Ln événeme'nt de probabilité suffisamment petite ne se produit jamais cou plus justement : nous devons agir comme sil ne devait pas se produire).

Cette seule règle suffit, parce qu'il est, cn efïcl, toujours possible de lier au phénomène étudié un événement de probabilité arbitraitement petite; si, à l'épreuve cet événement se produit néanmoins, il faudra admettre, en vertu de la loi unique du hasard, que les probabilités données qui ont été le point de départ du calcul ont été mal évaluées, ou même que le phénomène en question n'est pas aléatoire et qu'il ne relève pas de la théorie de la probabilité.

Or, cette loi, de nature évidemment extramathématique, est interprétée diversement par les subjectivistes et les objectivistes, mais tous l'admettent en définitive, et on le comprendra aisément, si nous l'illustrons, avec M. Boret, du célebre exemple de la dactylographe : une dactylographe ignorant lallemand, et isoléc avec sa machine, tape des lettres au hasard, el se trouve avoir, au bout de trois mois, tapé sans une variante le texte allemand des auves completes de Gothe; un tel «miracle », dont la probabilité est extrêmement faible, n'est pas, à strictement parler, impossible, mais toute persome de bon sens le tiendra pour pratiquement impossible, et si l'on nous disait qu'il a eu lieu, nous croirions fermement it une supercherie.

Il faut évidemment savoir quelle valeur la probabilité d'un événement ne doit pas dépasser pour qu'il soit à considérer comme « pratiquement impossible ». Cela dépend, naturellement, de l'échelle à laquelle on se place; mais pour une échelle donnée, M. Borre a montré que l'on peut évaluer l'ordre de grandeur de la valeur limite avec plus de précision qu'on no croirait, au premier abord; ainsi, à l'échelle humaine, c'est-à- 
dire pour les circonstances de la vie courante, une probabilité est négligeable qui est inférieure à $10^{-6}\left(10^{-6}\right.$ est à peu près la probabilité que nous avons d'être écrasés quand nous sortons dans les rues de Paris, risque que nous négligeons en effet). A l'échelle cosmique ou supercosmique, à laquelle doit se référer une théorie scientifique, la valeur limite devra être abaissée à $10^{-100}$ ou $10^{-200}$.

Mais si nul ne peut contester la légitimité de l'usage des mathématiques élémentaires ou transcendantes, dans toutes les branches de l'activité et des préoccupations humaines, et notamment en hydrologie, et les services qu'une telle discipline peut rendre, ici, comme en toutes choses, il faut, comme on dit : "Uti et non abuti », c'est-à-dire user et non abuser. Le bon sens a toujours son mot à dire, l'esprit de discernement doit toujours s'exercer parallèlement à l'esprit de rigueur, un juste équilibre doit être maintenu entre les préoccupations d'ordre hydrologique et les préoccupations d'ordre mathématique et spéculatif. Il ne faut pas, notamment, avoir recours à des calculs et à des formules dont la complication et la transcendance sont manifestement incompatibles avec la précision des données expérimentales qui les inspirent, ou avec l'efficacité des enseignements d'ordre pratique en vue desquels ces calculs sont faits et ces formules établies. Il ne faut pas, également, généraliser trop hâtivement, attribuer une portée générale à des interprétations mathématiques trop localisées dans le temps et dans l'espace. A quoi bon chercher à déterminer la $3^{\circ}$ décimale d'un nombre, lorsqu'on n'est même pas certain de la valeur de ses unités! Il faut également savoir se mettre à la portée de ceux qui vous lisent. "On a toujours tort d'admettre, à priori, a écrit DEDEBAND, que tout le monde sait de quoi on parle. Faule de mettre les points sur les $i$, la statistique est dans ses applications pleine de malentendus et l'on pourrait confectionner un assez beau collier avec toutes les perles pêchées chez les statisticiens d'honnête réputation. Ceux qui ont imaginé les méthodes se sont ordinaire- ment peu souciés d'en indiquer la valeur d'application; ceux qui les ont mises en œuvre les ont adoptées les yeux fermés, en se hâtant d'oublier les hypothèses et les réserves qui les restreignent.

Pour tout dire, il faut rester "hydrologue », hydrologue plus ou moins mathématicien, dans la mesure de ses moyens, et de ses goûts, et ne considérer les mathématiques non pas comme un but, mais comme un moyen, comme un langage, un bean langage certes, qui a sa beauté propre, mais dont la beauté ne doit pas faire oublier les faits et les idées que son ròle est d'exprimer et de traduire, sans les mutiler et les déformer.

Et je citerai, en terminant, à ce sujet, une page du grand botaniste Alp. DE CANDolle, extraite de son ouvrage de géographie botanique raisonnée, de 1855 :

«Parmi les méthodes dont on abuse, il faut citer en premier lieu l'emploi des chiffres. Il y a, dans chaque science, art ou objet d'étude, une partie statistique ou numérique. On la retrouve en agriculture, en médecine, dans toutes les branches de l'administration, dans les sciences physiques, naturelles et jusque dans les sciences morales. Elle occupe une très grande place dans la géographie botanique. Pour moi, j'en conviens, j'aime les chiffres autant que d'autres les détestent, mais ce qui me plaît, ce n'est pas d'accumuler des chiffres, c'est de montrer à quel degré il est nécessaire de choisir convenablement les valeurs, de les discuter, en d'autres termes de les subordonner aux lois de la logique et du bon sens qui dominent tout.

«Je me suis plu, dans bien des occasions, et même dans des sciences fort éloignées de la géographie botanique, à recueillir des chifrres de la manière la plus exacte, à les disposer le plus clairement possible, et aussi à discuter la portée des grands travaux de statistique mal compris dans le public ou de nature à servir d'exemples comme méthode. J'ai souvent pensé de la statistique ce qu'on dit de la parole : "Quid péjus, quid mélins.»

\section{II. - DISTRIBUTIONS STATISTIQUES ET COURBES DE PROBABILITÉ EXPÉRIMENTALES ET THÉORIQUES}

L'étude d'un phénomène, concrétisé par une variable statistique $(x)$ et considéré indépendamment de tous les facteurs qui le conditionnent, et indépendamment de l'ordre chronologique que suit son évolution, conduit naturellement - des qu'on cherche à dépasser la notion première, et élémentaire, évidemment trop simpliste de la moyenne, à la notion de fréquence, traduite par un diagramme, dit diagramme de fréquences. Un tel graphique dome le nombre d'observations correspondant à une valeur de $x$ comprise dans une série d'intervalles égaux $\Delta x$ successifs. Plus le nombre d'observations $N$ sera grand, plus l'intervalle $\Delta x$ pourra être choisi petit, et plus le 


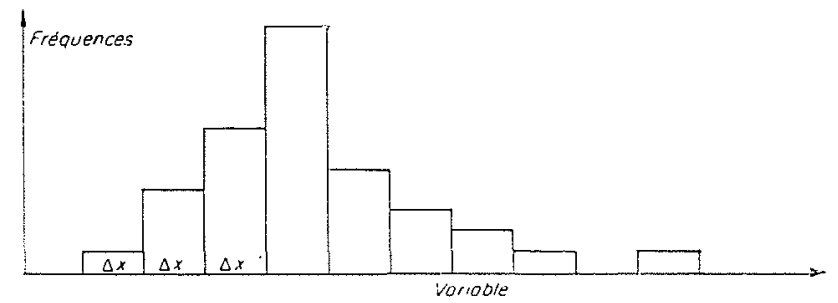

Fic. 1 .

diagramme pourra être assimilé à une courbe continue, ce qui n'est rigourcusement vrai que pour $\mathrm{N}$ infiniment grand et $\Delta x$ infiniment petit.

Cette courbe représente la distribution statistique de la variable $x$, supposée continue, et constitue ce qu'on appelle la courbe de probabilité.

Etant donné que dans la pratique courante des sciences expérimentales le nombre des ob-

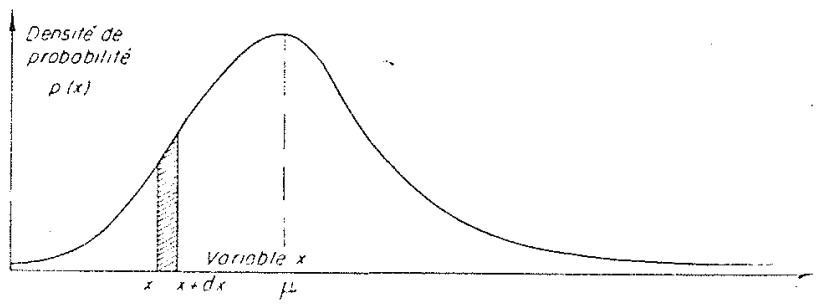

FiG. 2.

servations dont on peut faire état reste plus ou moins limité, le tracé de cotte courbe, s'il peut ètre considéré comme relativement exact, dans sa partie médiane, est plus ou moins indéterminé, dans ses parties extrêmes, dans le cas le plus général où la distribution doit être con-

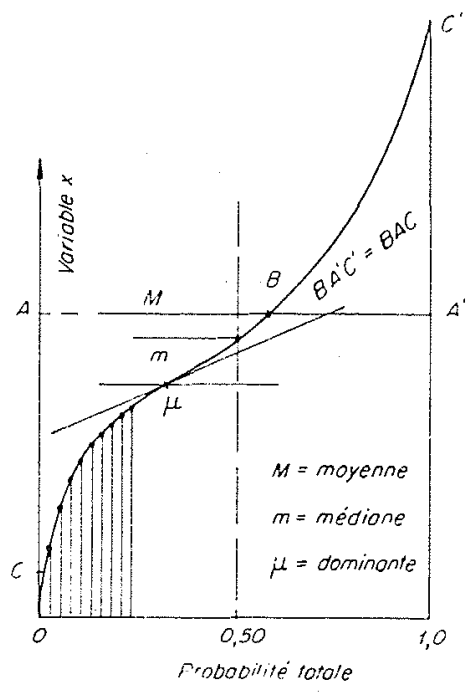

Fig. 3.

sidérée comme étant théoriquement illimitée dans les deux sens, c'est-à-dire le cas le plus fréquent où l'on ignore les limites maxima et minima de la variable $x$.
Le fait d'interpréter algébriquement, au moyen d'une équation thérique, une telle courbe, ce qui constitue une extrapolation, laisse naturellement subsister cette imprécision et cette indétermination.

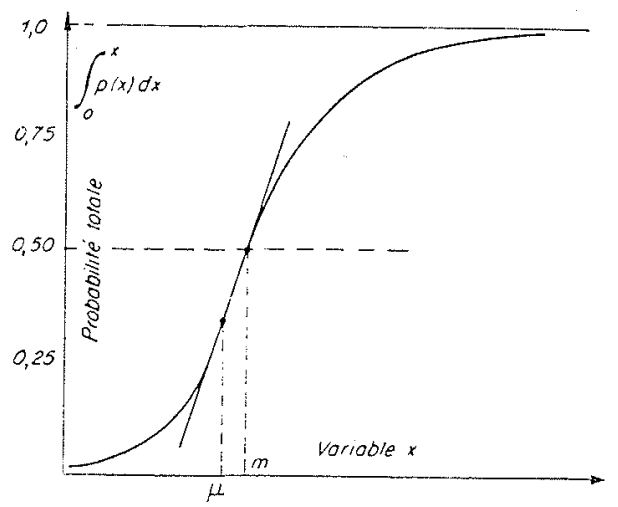

FIG. 4.

Quoi qu'il en soit de cette réserve, nous supposons l'existence d'une fonction $p(x)$, dite densité de probabilité, telle que $p(x) d x$ soit la probabilité pour que la variable $x$ soit comprise entre $x$ et $x+d x$.

Cette fonction $p(x)$ satisfait nécessairement la relation :

$$
\int_{-\infty}^{+\infty} p(x) d x=1
$$

qu'on appelle la condition de l'aire.

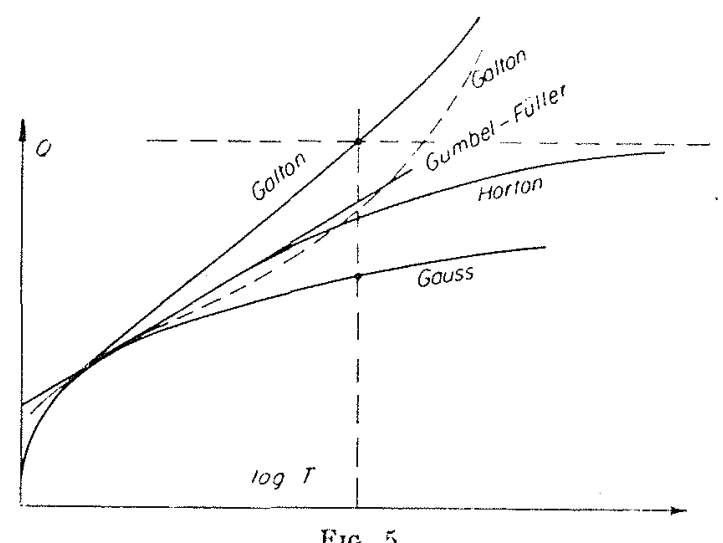

La probabilité dite totale d'une valeur $<x$ sera donnée par l'expression :

$$
\begin{gathered}
\int^{x} p(x) d x=\mathrm{P}(x) \text { en sorte qu'on a } \\
p(x)=\mathrm{P}^{\prime}(x)
\end{gathered}
$$

On construit souvent, notamment en hydrologie fluviale ( $x$ étant le débit journalier, le débit mensuel, ou tel autre débit) directement à partir 
des observations la courbe $P(x)$ qui exprime la probabilité totale de la variable $x$, cette courbe étant d'ailleurs présentée sous la forme $x=\varphi(P)$, en inversant les axes de coordonnées. Ainsi présentée, la courbe exprime le débit en fonction de sa probabilité totale $P$ : cette courbe n'est autre que l'enveloppe des débits classés par ordre de grandeur.

Elle est d'un usage fréquent, ancien et classique, en hydrologie fluviale où elle porte, suivant les auteurs et les pays, des noms variés : courbe de régime, courbe des durées, courbe des frécquences cumulées, etc.

En biologie, la courbe de probabilité elle-même, désignée souvent par courbe synoptique (du caractère $x$ étudié) est plus généralement employée; et cette pratique est, de certains points de vue, préférable, car elle met en relief certaines particularités (notamment l'existence de plusieurs maximas résultant du dimorphisme ou polymorphisme du phénomène, du caractère dont on étudie la variation), particularité que masque souvent la courbe $P(x)$ qui est une fonction qui ne diminue jamais et qui satisfait aux conditions $P(-\infty)=0$ et $P(+\infty)=1$ : cette dernière équation n'est autre qu'une autre forme de la condition de l'aire.

La probabilité d'une valeur $>x$ est évidemment $1-\mathrm{P}(x)$. Une distribution est dite symétrique, vis-à-vis de l'origine, si on a $p(x)=p(-x)$. On a évidemment dans ce cas :

$$
\mathrm{P}(-x)=1-\mathrm{P}(x) \text {. }
$$

\section{Transformation de la VARTable :}

Si la variable statistique $x$ est fonction d'une autre variable statistique $(t)$, soit $x=g(t)$, la distribution $\varphi(t)$ de la variable $t$ sera - puisque $p(x) d x=\varphi(t) d t$ :

$$
\stackrel{\varphi}{(t)}=p[g(t)] g^{\prime}(t)
$$

\section{VAleurs caractéristigues OU MOYENNES :}

On peut caractériser une distribution par certaines valeurs de la variable dites caractéristiques, dont les plus usuelles sont :

- La valeur médiane ou centrale $(m)$ définie par :

$$
\mathrm{P}(m)=1 / 2 ;
$$

- La valeur dominante ou mode (u.) qui est celle pour laquelle la densité de probabilité est maximum. Elle est définie par la relation :

$$
p^{\prime}\left(\mu_{0}\right)=0
$$

- La valeur moyenne arithmétique, dite aussi expérence mathématique définie par la relation :

$$
\mathrm{M}=\int_{-\infty}^{\infty} x p(x) d x
$$

En cas de distribution, limitée d'un côté ou de l'autre, on peut également considérer la valeur maximum $\left(M_{0}\right)$ et la valeur minimum $\left(m_{0}\right)$.

\section{ECARTS OU DÉviations}

Les différences entre une moyenne et chacune des observations sont appelées écarts ou déviations. Après avoir caractérisé une distribution par une moyenne, le problème se répète pour les écarts. Il faut en choisir un pour caractériser les déviations par rapport à la moyenne, c'està-dire pour savoir dans quelle mesure cette moyenne caractérise la distribution.

On définit l'écart central (e), dit aussi l'écart probable, comme tel que les probabilités de le dépasser ou non sont égales. Cette définition n'est possible que pour les distributions symétriques, pour les distributions asymétriques on fixe les valeurs de $x, x_{1}$, et $x_{2}$ telles que $\mathrm{P}\left(x_{1}\right)=1 / 4$ et $\mathrm{P}\left(x_{2}\right)=3 / 4$ et $x_{1} \leqslant x \leqslant x_{2}$ définit l'intervalle probable.

Une autre mesure des écarts est la moyenne des valeurs absolues des écarts, calculée à partir de l'espérance mathématique ou moyenne. On l'appelle l'écart moyen $(\theta)$. Il faut bien la distinguer de la moyenne des écarts qui sera toujours nulle quand on prend comme point de départ la moyenne arithmétique.

L'écart moyen $\theta$ pour une distribution continue est défini par la relation :

$$
0=\int_{-\infty}^{+\infty}(x-\mathrm{M}) p(x) d x=2 \int_{-\infty}^{\mathrm{M}}(\mathrm{M}-x) p(x) d x
$$

On définit également l'écart-type ( $\sigma$ ) ou écart quadratique moyen, par la racine carrée de la valeur probable du carré de l'erreur, laquelle n'est pas égale à la valeur probable de l'erreur :

$$
\sigma^{2}=2 \int_{0}^{+\infty}(x-M)^{2} p(x) d x
$$

On appelle $\sigma^{2}$ la dispersion. Elle est égale au moment de rang 2 autour de l'espérance mathématique. Les deux premiers moments (de rang 0 et 1) sont la condition de l'aire et la définition de l'espérance mathématique. 
INTERPRETATION ALGEBBRIQUE D'UNE DISTRIBUTION STATISTIQUE:

Une distribution statistique résultant d'une série d'observations en nombre suffisant peut être interprétée et par suite extrapolée en dehors des limites de l'observation par une formule algébrique théorique.

De telles formules sont nombreuses, depuis la courbe élémentaire classique dite de GavssLaplace jusqu'aux formules variées de Pearson et autres mathématiciens statisticiens modernes. Leur nombre est variable à l'infini. Nous nous contentons ici d'indiquer et de caractériser les plus simples et les plus usuelles, dont l'emploi suffit généralement pour linterprétation des statistiques hydrologiques.

\section{Distribution de Gauss-Laplace}

Bien que la formule de Gauss, qui est celle qui caractérise la distribution statistique des erreurs accidentelles, soit archi-connue, il ne sera peutêtre pas inutile d'en rappeler les caractéristiques essentielles et les modalités pratiques de son emploi, notamment en hydrologie, que beaucoup d'hydrologues ignorent :

La formule est :

$$
p(x)=\frac{h}{\sqrt{\pi}} e^{-h_{i 2}^{2}\left(x^{-}-x_{0}\right)^{2}}
$$

Elle est symétrique et les trois moyennes cidessus définies sont égales :

$$
\mathrm{M}=m=\mu_{0}=x_{0}
$$

Effectuons le changement de variable :

$$
z=h\left(x-x_{0}\right) .
$$

On a :

$$
d z=h d x .
$$

La formule devient :

$$
p(z)=\frac{1}{\sqrt{\pi}} e^{-z^{2}}
$$

et on a par suite :

$$
\mathrm{P}(z)=\frac{1}{\sqrt{\pi}} \int^{z} e^{-\tilde{x}^{2}} d z
$$

Toutes les courbes de probabilité Gauss-LAPLACE se déduisent les unes des autres par transformation linéaire et, par suite, de la précédente. Il suffit donc, une fois pour toutes, de tracer cette courbe, on mieux, d'établir le barime des valeur's respectives de $\mathrm{P}$ et $z$ (que donnent tous les traités classiques), pour pouvoir étudier telle distribution statistique que l'on voudra particulière, susceptible d'être interprétée par une formule de GaUss.

Indiquons quelques valeurs respectives de $P$ et $z$ :

$$
\begin{aligned}
\mathrm{P}: 1 & \%-5 \%-10 \%-15 \%-20 \%- \\
& 25 \%-30 \%-35 \%-40 \%-45 \% \\
& -50 \% . \\
z:- & 1,648-1,166-0,907-0,739-0,596 \\
& -0,477-0,371-0,272-0,179-0,086 \\
& -0 .
\end{aligned}
$$

En changeant le signe de $z$, on aura les valeurs correspondantes $(1-\mathrm{P})$.

Pour voir si une distribution statistique $z_{1}, z_{2} \ldots z_{n}$ pent être interprétée par une formule de Gauss, et pour déterminer, dans ce cas, graphicquement notamment, ses caractéristiques $(h)$ et $x_{0}\left(x_{0}=\mathrm{M}\right)$, il suffit de situer, dans le plan, les points $x_{1} z_{1}, x_{2} z_{2} \ldots x_{n} z_{n}$, les axes des coordonnées étant gradués suivant les valeurs de $x$ (valeurs observées de la variable) et les valeurs de la variable réduite $z$.

$\mathrm{Si}$ les points sont suffisammeent en ligne droite, l'équation de cette droite étant :

$$
z=h\left(x-x_{0}\right)
$$

soit encore :

$$
x=x_{0}+\frac{z}{h},
$$

caractérise la distribution considérée comme gaussienne.

Par exemple, les valeurs de probabilité $25 \%$ et $75 \%$ soit $z_{1}$ et $x_{2}$, seront données par les égalités :

$$
x_{1}=x_{0}-\frac{0,477}{h} \quad x_{2}=x_{0}+\frac{0,477}{h}
$$

La valeur de $x$, dont la probabilité de dépassement est de $1 \%$, sera :

$$
x=x_{0}+\frac{1,648}{h}
$$

On peut également, après vérification graphique de l'alignement convenable des points $x_{n} z_{n}$, calculer $h$ par la considération des écarts, la valeur $x_{0}=\mathrm{M}$ (moyenne) étant prise égale à $\frac{\sum x}{\mathrm{~N}}$.

Nous rappelons les formules classiques des écarts dans la distribution de Gauss (E désigne un écart par rapport à la moyenne). 
Ecart moyen :

$$
\theta=\frac{1}{h \sqrt{\pi}}
$$

Ecart quadratique ou écart-type :

$$
\sigma=\sqrt{\frac{\Sigma \varepsilon^{2}}{n-1}}=\frac{1}{h \sqrt{2}}
$$

Ecart probable :

$e=\frac{0,47694}{h}=0,6745 \sqrt{\frac{\Sigma \varepsilon^{2}}{n-1}}[0,6745=0,47694 \sqrt{2}]$

L'emploi d'une de ces formules permet de calculer le facteur $h$ qui caractérise, avec la moyenne $x_{0}=\mathrm{M}$, la distribution :

$$
h=\frac{0,47694}{e} \quad h=\frac{1}{0 \sqrt{ } \pi} \text { ou } h=\frac{0,56419}{0}
$$

On peut également, pour une détermination rapide de $h$ et $\mathrm{M}$, considérer seulement deux points particuliers, par exemple ceux correspondant aux valeurs $x_{1}$ et $x_{2}$ de probabilité $25 \%$ et $75 \%$ (dits quartils).

Des deux équations :

$$
\begin{array}{r}
0,47694=h\left(x_{2}-\mathrm{M}\right) \\
-0,47694=h \cdot\left(x_{1}-\mathrm{M}\right)
\end{array}
$$

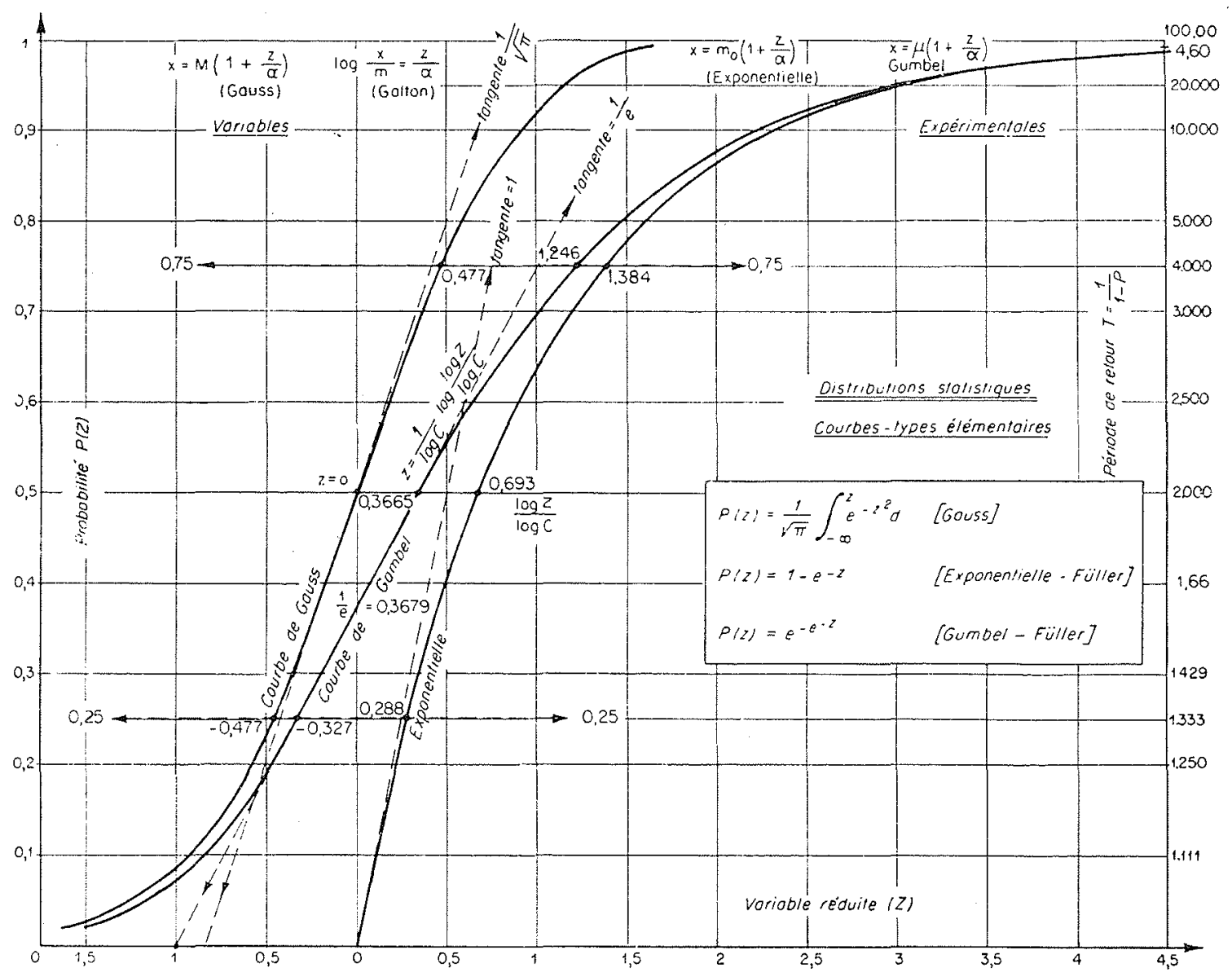

FYG 6.

on tire :

$$
\mathrm{M}=\frac{x_{2}+x_{1}}{2}
$$

et :

$$
h=\frac{0,95388}{x_{2}-x_{1}}
$$

Plus la distribution sera gaussienne, plus les valeurs ainsi trouvées pour $\mathrm{M}$ et $h$ seront voisines.

Indiquons seulement les deux relations suivantes, d'autant plus exactes que la distribution est plus gaussienne:

$$
\frac{2 \mathrm{~N} \mathrm{\Sigma}^{2}}{[\mathrm{\Sigma} \mid \mathrm{\varepsilon}]]^{2}}=\pi \quad \text { et } \quad \frac{\theta}{e}=1,183
$$


et également la répartition théorique des écarts par rapport à l'écart probable pour $\mathrm{N}=10.000$ :

$$
\left\{\begin{array} { l l } 
{ \varepsilon > \frac { e } { 2 } } & { 7 . 3 5 9 } \\
{ \varepsilon > e } & { 5 . 0 0 0 } \\
{ \varepsilon > 2 e } & { 1 . 7 7 4 }
\end{array} \left\{\begin{array}{lr}
\varepsilon>3 e & 430 \\
\varepsilon>4 e & 70 \\
\varepsilon>5 e & 7
\end{array}\right.\right.
$$

Notons enfin que l'on peut écrire la formule caractéristique de la distribution de Gauss sous la forme :

$$
\frac{x}{\mathrm{M}}=1+\frac{z}{\mathrm{~K}}
$$

$\mathrm{K}=h \mathrm{M}$

Cette forme est particulièrement intéressante à considérer dans les études comparatives, entre régions et bassins différents : $\mathrm{K}$ caractérise la variabilité spécifique de la distribution, c'est-àdire la variabilité de $x$ en prenant comme unité la valeur moyenne $M$.

\section{Distribution logarithMiQue de Gatton (Loi de l'effet proportionnel)}

Pour qu'une variable ait une distribution statistique symétrique, et, en particulier, gaussienne, il faut que ses possibilités de variations extrèmes de part et d'autre de la valeur moyenne soient égales, en d'autres termes que le champ de ses variations théoriquement possibles s'étende de $-\infty \dot{a}+\infty$.

C'est là une condition qui n'est, pour ainsi dire, jamais réalisée exactement. Toutes les courbes de probabilités en météorologie, climatologie et hydrologie sont plus ou moins dissymétriques.

Si le champ de variations ne peut varier que de 0 (ou de toute autre valeur finie, positive ou négative $\left(x_{0}\right)$ à $+\infty$, c'est son logarithme log $x$ [éventuellement $\log \left(x-x_{0}\right)$ ], dont le champ de variations s'étend de $-\infty$ d $+\infty$, qui pourra éventuellement suivre la loi de Gauss.

Cette simple remarque élémentaire dictée par le bon sens, suffit à suggérer et à légitimer la distribution caractérisée par les relations :

$$
\begin{aligned}
\mathrm{P}=\frac{1}{\sqrt{\pi}} \int_{-\infty}^{\approx} e^{-z^{2}} d z \text { et } z & =a \log x+b, \text { ou }: \\
z & =a \log \left(x-x_{0}\right)+b
\end{aligned}
$$

Cette distribution, dont a fait état pour la première fois, semble-t-il, GalTon (Philosophical Magazine, $4^{\circ}$ série, volume 49, 1875), et que nous désignons, pour cette raison, par Distribution ou formule de GatTon, a été utilisée avec succès, en hydrologie fluviale, pour l'interpréta- tion des courbes de probabilité des débits, par Hans Grassberger pour le Danube (L'emploi du Calcul des Probabilités dans l'étude des cours d'eau, die Wasserwirtschaft, 15 janvier 1932 et $\mathrm{n}^{\circ \text { s }}$ suivants), et par Gronat pour la Truyère (sur l'Ajustement mathématique des Courbes de débit d'un cours d'eau : C.R. Académie des Sciences, 7 mars 1932). Cel ingénieur l'a désignée sous le nom de «Loi de l'effet proportionnel», compte tenu de la remarque suivante :

Si les valeurs de $x$ suivaient la loi des erreurs fortuites, la variation $d x$ produite par une cause quelconque serait la même, quel que soit $x$, donc indépendante de $x$. Si cette variation $d x$ est proportionnelle à $\left(x-x_{0}\right)$, le rapport $\frac{d x}{x-x_{0}}$ jouera le même rôle que la variation élémentaire dans la loi des erreurs, et, par suite, la quantité obtenue par intégration, soit $a \log \left(x-x_{0}\right)+b$, suivra la loi des erreurs.

Cette distribution est caractérisée par les valeurs moyennes suivantes :

Valeur médiane $(m)$. - Elle est donnée en faisant $z=0$, d'où son expression :

$$
\log \left(m-x_{0}\right)=-\frac{a}{b} .
$$

En mettant en relief cette valeur médiane, l'expression $z=a \log \left(x-x_{0}\right)+b$ s'écrit :

$$
z=a \log \left[\frac{x-x_{0}}{m-x_{0}}\right]
$$

oll si :

$$
x_{0}=0 \quad z=a \log \left(\frac{x}{m}\right)
$$

ou encore :

$\log \frac{x}{m}=\frac{z}{a}$, formule correspondant à la formule $\frac{x}{\mathrm{M}}=1+\frac{Z}{a}$. de la distribution de Gauss.

On remarquera qu'une distribution étant interprétée par une distribution de Gauss :

$$
\frac{x}{\mathrm{M}}=1+\frac{z}{a^{\prime}}
$$

et par une distribution de GaLton, on a théoriquement, donc approximativement, $a^{\prime}=a \log e$, relation qu'on obtient en développant en série :

$$
\frac{x}{m}=e^{\frac{z}{a \log e}}=1+\frac{z}{a \log e}+\ldots e
$$


en négligeant les termes au-delà du deuxième, d'autant plus négliggeables que $a$ est plus grand et $z$ plus petit (partie centrale de la distribution); et en admettant $m=M$.

Valeur dominante (u). - Elle s'oblient en annulant la dérivée seconde de $\mathrm{P}$ (point d'inflexion) considérée comme fonction de $x$.

$$
\mathrm{P}^{\prime}(x)=e^{-z^{2}} z^{\prime} \quad \mathrm{P}^{\prime \prime}=e^{-z^{\prime 2}}\left[z^{\prime \prime}-2 z z^{\prime 2}\right]=0
$$

D'où :

$$
\begin{aligned}
z=-\frac{1}{2 a \log e}=a \log \left(u-x_{0}\right)+b \\
\qquad \log \left(\mu-x_{0}\right)=-\frac{b}{a}-\frac{1}{2 a^{2} \log e}
\end{aligned}
$$

ou encore :

$$
\log \left(u-x_{0}\right)=\log \left(m-x_{0}\right)-\frac{1}{2 a^{2} \log e}
$$

Valeur moyenne, - On calcule M par la formule :

$$
\mathrm{M}-x_{0}=\int_{-\infty}^{+\infty}\left(x-x_{0}\right) p(x) d x
$$

On obtient la formule :

$$
\begin{aligned}
\log \left(\mathrm{M}-x_{0}\right)=-\frac{a}{b} & +\frac{1}{4 a^{2} \log e} \\
& =\log \left(m-x_{0}\right)+\frac{1}{4 a^{2} \log e}
\end{aligned}
$$

On notera la relation :

$$
\left(m-x_{0}\right)^{3}=\left(\mu-x_{0}\right)\left(\mathrm{M}-x_{0}\right)^{2}
$$

entre les trois moyennes $m$, y. et M.

Entre deux valeurs de probabilités complémentaires $x^{\prime}$ et $x^{\prime \prime}$, on a la relation :

$$
\left(x^{\prime}-x_{0}\right)\left(x^{\prime \prime}-x_{0}\right)=\left(m-x_{0}\right)^{2}
$$

soit :

$$
\sqrt{x^{\prime} x^{\prime \prime}}=m\left(\text { si } x_{0}=0\right)
$$

formule correspondant à la relation :

$$
\frac{x^{\prime}+x^{\prime \prime}}{2}=m
$$

de la formule de Gauss. On peut rapidement, au moyen de ces deux formules, se rendre compte rapidement, en première estimation, de la nature, gaussienne ou galtonienne, d'une distribution.

Plus exactement, pour interpréter une distribution expérimentale au moyen de la loi de GaL-
TON et en déterminer ses caractéristiques $a, m$ (et éventuellement $x_{0}$ ), on procédera exactement comme nous l'avons indiqué poux une distribution gaussienne, $\log x$ ou $\log \left(x-x_{0}\right)$ remplacant la variable $x$.

On admettra d'abord $x_{0}=0$. Si l'alignement rectiligne des points n'est pas très satisfaisant, si la courbe $(z, \log x)$ est plus ou moins incurvée dans un sens ou dans l'autre, on déterminera par tâtonnements successifs la valeur $x_{0}$ (positive ou négative) dommant l'alignement rectiligne le meilleur.

L'équation de la droite ainsi obtenue :

$$
z=a \log \frac{x-x_{0}}{m-x_{0}}
$$

caractérisera la distribution au moyen des trois paramètres $a, m$ et $x_{0}$.

Il faut noter, d'ailleurs, que le choix de la constante dite d'ajustement $x_{0}$ est plus ou moins subjectif, plus ou moins indéterminé, done conventionnel, et que des valeurs voisines de $x_{0}$ différentes, conduisent souvent à des alignements aussi satisfaisants à première vue les uns que les autres, et entre lesquels il est difficile de choisir le meilleur.

La valeur du paramètre a qualifie la variabilité et la dissymétrie de la distribution, d'autant plus grande que ce coefficient est plus petit. Plus il est grand, plus $\frac{1}{a^{2} \log e}$ est petit, et plus, par conséquent, sont voisines les valeurs $M, m$ et $\mu$, qui tendent à s'égaliser au fur et à mesure que la distribution devient plus gaussienne. A partir de $a=10$, on peut considérer la distribution comme telle, tout au moins entre certaines limites assez étendues de probabilité.

La dissymétrie de la distribution peut être également chiffrée par le rapport $\mathrm{M} / \mathrm{m}$. Théoriquement, les valeurs de $\mathrm{M} / \mathrm{m}$ et de a se correspon. dent comme il suit, suivant la formule :

$$
\log \frac{M}{m}=\frac{1}{4 a^{2} \log e}
$$

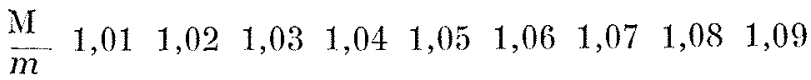

$\begin{array}{llllllllll}\text { a } & 11,55 & 8,18 & 6,70 & 5,80 & 5,20 & 4,77 & 4,08 & 4,15 & 3,92\end{array}$

$$
\begin{array}{lll}
\frac{\mathrm{M}}{\mathrm{m}} & 1,10 & 1,11 \\
\text { a } & 3,73 & 3,53
\end{array}
$$

Comme exemple de cette distribution, citons celle du débit journalier de la Truyère qui, d'après les calculs de Grbratr, est définic par la relation :

$$
z=2,027 \log (\mathrm{Q}+4)-2,87
$$


Elle donne lieu aux comparaisons suivantes : M observé ....... 30,0 M calculé 29,5 $m \ldots \ldots \ldots \ldots \ldots \quad 19,6 m \ldots \ldots 20,3$ $\begin{array}{lllll}2 \ldots \ldots \ldots \ldots & 9,0 & \ldots & \ldots \ldots & 8,7\end{array}$

Max. caractéristique... $\quad 110,0 \ldots \ldots \quad 109,0$

Min. caractéristique... .

$2,4 \ldots \ldots \ldots$

Rapport $\frac{M}{m} \ldots \ldots \ldots=\frac{19,5}{30}=1,54$

Rapport calcule .... $=\frac{29,5}{20,0}=1,45$

On notera incidemment que la formule ne convient absolument pas pour les très faibles débits. Et c'est là une preuve qu'il est bien difficile, pour ne pas dire impossible, d'interpréter, au moyen d'une seule formule, tout le champ des variations d'une variable telle que le débit d'un cours d'eau : les variations des débils extrèmes sont conditionnées différemment, celles des faibles débits par la capacité de rétention du sol, celles des forts débits par celles des fortes précipitations. Le ròle des capacités de rétention nivale et glaciaire produit des effets analogues sur les courbes de probabilité correspondantes, trop complexes pour qu'on puisse les interpréter fidèlement par une seule courbe théorique. Il y a intérêt, dans ce cas, à considérer deux courbes distinctes, l'une relative au semestre hivernal, l'autre au semestre estival, par exemple.

\section{DISTRIBUTION EXPONENTIELLE}

Pour mémoire, nous mentionnons, ici, rapidement, cette distribution que l'on rencontre, en hydrologie, comme limite de la distribution de Gumbel, dont nous parlerons plus loin.

Elle est définie par les relations suivantes :

$$
\begin{array}{ll}
p(z)=e^{-z} & p(x)=\alpha e^{-a\left(x-x_{0}\right)} \\
z=\alpha\left(x-x_{0}\right) & \mathrm{P}(x)=1-e^{-a\left(x-x_{0}\right)}
\end{array}
$$

Valeur médiane : $e^{-\cdots, z}=1 / 2$,

$$
\text { donc : } \quad m=x_{0}+\frac{\log 2}{\alpha \log e}
$$

Valeur moyenne :

$$
\mathrm{M}=\int_{-\infty}^{+\infty} z e^{-\%} \quad \mathrm{M}=x_{0}+\frac{1}{\alpha}
$$

Valeur dominante : $\mu=x_{0}$ (valeur minimum).
La probabilité d'une valeur $>z$ est :

$$
\mathrm{I}-\mathrm{P}(z)=e^{-\tilde{z}}
$$

Désignons cette probabilité par $\frac{1}{\mathrm{~T}}$ (T désigne ce qu'on appelle souvent la période de retour).

On en déduit :

$$
\frac{1}{\mathrm{~T}}=e^{-z}
$$

$\log \mathrm{T}=z \log e$

$$
\log \mathrm{T}=\alpha\left(x-x_{0}\right) \log e
$$

$$
x=x_{0}+\frac{\log \mathrm{T}}{\alpha \log e}
$$

ou encore, finalement :

$$
x=x_{0}\left[1+\frac{\log \mathrm{T}}{\approx x_{0} \log e}\right]
$$

Il y a, entre $x$ et $\log \mathrm{T}$, une relation linéaire. Nous retrouverons plus loin celte formule, particulièrement intéressante à considérer dans l'étude des crues.

\section{DISTRIBUTION DOUBLEMENT EXPONENTIELLE DE GUMBEL}

(loi de la plus grande valeur)

On me permettra de faire un bref exposé historique au sujet de cette formule, dont j’ai été le premier à signaler l'intérêt en hydrologie, notamment dans l'étude des crues, et à montrer l'identité qu'elle présente avec la formule exponentielle, à partir d'une certaine probabilité, c'està-dire avec la formule dite de Fuluer.

Cette loi, dite de la plus grande valeur, a été formulée par le mathématicien allemand GumBEL, à la suite de ses études sur la distribution statistique " des plus grands âges ». Ayant eu l'occasion, lor's d'une collective à skis, de faire sa connaissance (proserit par le régime nazi, il s'était réfugié à Lyon, et y avait trouvé une place comme assistant à l'Université de Lyon, Institut des Sciences financières et d'assurances) et de causer avec lui « mathématiques », je lui suggérai que sa formule devait être applicable au cas des crues, c'est-à-dire «des plus grands débits», problème analogue à celui des plus grands âges. L'expérience confirma cette suggestion et la formule se montra satisfaisante, dans l'interprétation des statistiques de crue d'un certain nombre de cours d'eau français (le Rhône, le Rhin, la Cère) et américains (le Merrimack, le Tennessee, le Mississipi), peut-être pas plus satisfaisante, 
mais autanl que la formule de GaLTon, expérimentée concurremment. J'ai présenté alors, c'était en 1936, deux communications à la Société Hydrotechynique de France, à ce sujet, et provoquai, au sein de cette Société, une réunion où MM. Gibrat et Gumber échangèrent leurs idées.

En 1989, M. Gumber s'est réfugié à New-York et a répandu sa formule aux Etats-Unis, où elle est aujourd'hui d'un usage courant, officiellement adoptée notamment par le Geological Survey, l'Ohio Water Resources Board, etc. Dans l'ouvrage : Floods of May June 19.8 -- Columbia River Basin, du Geological Survey, water supply paper 1080-1949, le régime des crues d'une centaine de bassins de la Columbia river est synthétisé par des graphiques interprétant la distribution statistique des plus forts débits annuels, au moyen de la formule de Gumbl. Cette interprétation apparaît, d'après ces graphiques, digne de retenir lattention.

L'ouvrage récent publié à New-York en 1949: Applied Hydrology, de Linsles, KoHLEn et PAvLHus, fait mention de celte loi et en donne une application concernant les crues de la KansasRiver.

Cette distribution est caractérisée par les formules suirantes :

$$
\begin{aligned}
& p(y)=e^{-y \cdots e^{-y}} \\
& P(y)=e^{-y} \\
& y \quad=\alpha\left(x-e^{-y}\right)
\end{aligned}
$$

$y$ joue ici un ròle analogue à celui de la variable réduite $z$ de la distribution de Gavss, ou de la distribution exponentielle. Quant an parametre $\mu$, c'est la valeur dominante de la distribution.

Le coefficient de variabilité $\%$ se déduit de l'écart-type $\sigma$ par la formule :

$$
\sigma^{2}=\frac{\pi^{2}}{6 x^{2}}
$$

soit $1 / \sigma=0,77968 \sigma ;$ la valeur moyenne $M$ et la valeur médiane $m$ sont données respectivement par les formules :

$$
\begin{aligned}
& \mathrm{M}=\mu+\frac{0,577216}{\alpha} \\
& m=\mu+\frac{0,3665}{\alpha}
\end{aligned}
$$

On a également la formule $\mu=M-0,450005$ o entre l'écart-type et la valeur dominante p. ct la formule $1 / x=0,63591\left(x_{2}, \cdots, x_{1}\right)$ entre $z$ et la différence des valeurs de probabilités $25 \%$ et $75 \%$.

Comme pour la variable de Gauss, des tables donnent les valeurs de $y$ et de P se correspondant et pour expérimenter cette formule, el en calculer les paramètres $\alpha$ et $u$, on procédera exactement comme indiqué plus haut, soit graphiquement, soit en utilisant l'une des formules ci-dessus.

On remarquera que pour $y=0(x=\mu=$ valeur dominante) on a : $\mathrm{P}=e^{-1}=\frac{1}{e}=0,36788$. La probabilité totale de la valeur dominante (égale à 0,50 dans la distribution de Gauss) est ici égale à $\frac{1}{e}=0,36788$.

Le rapport entre l'écart-type et l'écart moyen (iggal à $\sqrt{\frac{\pi}{2}}=1,25331$ dans la distribution de Guuss) est égal à 1,30593.

Enfin la distribution tend très rapidement vers la distribution exponentielle avec laquelle elle s'identific pratiquement à partir de $\mathrm{P}=0,99$ $(y=4,60)$; en effet, si nous désignons par $\varepsilon$ l'expression de $e^{-x}$, on a :

$$
P(z)=e^{-\varepsilon=1-\varepsilon+\frac{\varepsilon^{2}}{2} \cdots}
$$

Pour P suffisamment grand, on peut négliger les termes de la série au-delà du $2^{\text {c et }}$ on retrouve la formule exponentielle simple :

$$
\mathrm{P}=1-e^{-z} \quad x=u \cdot\left[1+\frac{\log \mathrm{T}}{\alpha \mu \log e}\right]=u \cdot[1+\mathrm{K} \log \mathrm{T}]
$$

C'est la formule ancienne de Füldxr, établic par lui expérimentalement, valable seulement pour ' $T$ suffisamment grand. La formule de GumBEL Ia légitime mathématiquement, la complète pour les faibles valeurs de $P$ et précise que la moyenne à considérer, en l'occurrence, n'est pas la moyenne arithmétique $\mathrm{M}$, comme le concevait, semble-t-il, Fülter, mais la valeur dominante $y$.

\section{FORMULES DIVERSES}

Distribution constante. -... Une distribution est dite limitée dans les deux sens, lorsque la variable statistique reste comprise entre deux valeurs limites $x_{m}$ et $x_{0}$ parfaitement définies. Le cas, théorique, le plus simple que l'on puisse concevoir est celui d'une distribution constante, dans laquelle toutes les valeurs sont dominantes, en ce sens qu'il n'en existe pas.

La courbe de probabilité se présente comme un rectangle :

$$
\begin{aligned}
& p(x)=\alpha=\frac{1}{x_{m}-x_{0}} \\
& P(x)=x_{0}+\frac{p}{x}=\frac{x-x_{0}}{x_{m}-x_{0}}
\end{aligned}
$$


La valeur médiane et la valeur moyenne sont égales :

$$
\mathrm{M}=m=\frac{x_{0}+x_{m}}{2} .
$$

L'écart moven :

$$
0=\frac{x_{m}-x_{0}}{4}=\text { l'écart probable. }
$$

La dispersion est :

$$
\sigma^{2}=\left(x_{m}-x_{0}\right)^{2}\left[\frac{1}{3}-\frac{1}{4}\right]=\frac{\left(x_{m}-x_{0}\right)^{2}}{4}
$$

On peut considérer la distribution de Gauss comme étant la résultante d'une infinité de distributions constantes.

D'une manière imagée, on peut dire que la distribution de GaUss est une distribution constante déformée par le hasard.

Distribution logarithmigue constante. Elle se déduit de la précédente en remplaçant la variable par son logarithme.

On a $: p(x)=\frac{\alpha}{x}$ : la densité de probabilité est inversement proportionnelle à la variable :

$$
\begin{gathered}
\alpha=\frac{1}{\mathrm{~L} x_{m}-\mathrm{L} x_{0}}=\frac{\log e}{\log x_{m}-\log x_{0}} \\
\mathrm{P}(x)=\int \frac{\alpha}{x}=\alpha\left[\mathrm{L} x-\mathrm{L} x_{0}\right]
\end{gathered}
$$

La valeur moyenne est :

$\mathrm{M}=\int_{x_{0}}^{v_{m}} x p(x) d x=\alpha\left(x-x_{0}\right)=\alpha x_{0}\left[e^{1 / a}-1\right]$

La valeur médiane est donnée par la formule :

$$
\frac{\mathrm{L} x-\mathrm{L} x_{0}}{\mathrm{~L} x_{m}-\frac{\mathrm{L} x_{0}}{2}=1 / 2} \mathrm{~L} \frac{m}{x_{0}}=\frac{1}{2 \alpha} \quad \frac{m}{x_{0}}=e^{\mathrm{t} / 2 a}
$$

La distribution de GaLToN est à cette distribution ce qu'est la distribution de GuUss à la distribution constante. Ou encore : la distribution de Gabton est une distribution logarithmique constante, déformée par le hasard.

Dans les deux distributions, on a $x_{1} x_{2}=m^{2}$, $x_{1}$ et $x_{2}$ étant les valeurs de probabilité complémentaire.

Formule de Horton. - Partant de ce principe que toute distribution est, dans la pratique courante, limitée, Horton a proposé pour la statistique des débits de crue, par exemple, la formule :

$$
x=\mathrm{M}_{0}\left[1-e^{-\mathrm{KT} \mathrm{T}^{n}}\right]
$$

'T désignant, comme indiqué plus haut, la période de retour :

$$
\left(1-\mathrm{P}=\frac{1}{\mathrm{~T}}\right)
$$

C'est là une formule tout à fait empirique, sans valeur statistique, ne relevant pas du Calcul des Probabilités. Limitée supérieurement, la distribution se trouve également limitée inférieurement, la valeur minimum $m_{0}$ étant égale à :

$$
\mathrm{M}_{0}\left[1-e^{-\mathrm{K}}\right]
$$

Formule du type paraboligue. - Il en est de même de celle que nous avons proposée autrefois, qui, faisant abstraction des plus faibles et des plus forts débits, caractérise la courbe des débits classés d'un point de vue purement pratique et industriel :

$$
\frac{\mathrm{Q}-q_{0}}{\mathrm{M}-q_{0}}=(n+1)\left(\frac{x}{\mathrm{~T}}\right)^{n}=(n+1) \mathrm{P}^{n}
$$

en exprimant le débit en fonction du débit moyen $M$ d'un débit $q_{0}$, voisin du débit minimum absolu (qu'on ignore), et de la probabilité P. Le débit maximum se trouve également limité à une valeur $\mathrm{M}_{0}$ plus ou moins fictive, c'est-à-dire à une valeur de débit dont la probabilité de dépassement est de l'ordre de 1 à $3 \%$ par exemple.

Formules type Pearson. - A l'opposé de ces deux dernières formules, d'autres formules ont été proposées el expérimentées, plus complexes, pour interpréter et interpoler les courbes de fréquence, notamment en hydrologie. Elles ont été plus ou moins inspirées par les travaux de Pearson.

Fondées sur les considérations moins élémentaires que celles dont nous venons de parler, nous ne ferons que les signaler, en citant les études de Foster (courbes théoriques de fréquence 1924), d'Eudrenton (courbes de fréquence et corrélation, 1927), de HAZEN (Flood Flows, 1930), de Srade (une fonction asymétrique de probabilité, 1936), etc.

Plus récemment, MM. Le Cam et Mordat ont également utilisé certaines des courbes de Pr.rison. (Lois de probabilité des débits des cours d'eau. Travaux de la S.H.F., la Houille Blanche, 1949.)

\section{Représentation GRAPHIQUE}

des Courbes de probabilité - Levr comparaison

On remarquera que les courbes de probabilité théoriques que nous avons étudiées expriment la variable $x$ (en particulier le débit) en fonction de 
leur fréquence ou probabilité, d'un paramètre qualifiant la variabilité, et d'une valeur moyenne qui est :

a) Dans la distribution de Gauss, la valeur moyenne M (qui est d'ailleurs également la valeur médiane et la valeur dominante);

b) Dans la distribution de GaLTon, la valeur médiane $m$;

c) Dans la distribution de Gumber, la valeur dominante;

d) Dans la formule exponentielle, la valeur minima $m_{0}$;

e) Dans la formule de Horton, la valeur maxima $\mathrm{M}_{0}$.

Graphiquement, une distribution statistique peut être représentée de différentes manières, suivant la variable choisie comme exprimant la probabilité.

a) Par la courbe de la probabilité elle-même, la variable exprimant la probabilité étant la densité de probabilité;

b) Par la courbe de la probabilité totale $x=f(\mathrm{P})$ ou $\mathrm{P}=\rho(x)$;

c) Par la droite d'alignement des points représentés par les valeurs de la variable et les valeurs des variables réduites de la distribution adoptée;

d) Enfin, par la courbe $x=\mathrm{F}(\mathrm{T})$ exprimant la variable en fonction de la période de retour $\mathrm{T}$ comme elle a été définie plus haut.

Cette deniere méthode, quand il s'agit de comparer les différentes formules thériques dans la zone, non atteinte généralement par l'expérimentation, des faibles probabilités de dépassement, est particulièrement indiquée. Plus particulièrement, on considérera, non par. $T$, mais $\log \mathrm{T}$.

La partie de la courbe que l'on peut considérer comme à peu près déterminée par l'observation, étant, par exemple, $A B$, sera interpolée, dans la distribution de GaUss, par la courbe B C, la courbe totale A B C ayant une allure parabolique.

La courbe Gumbel se présentera comme prolongeant l'arc $A B$ par une tangente $B C^{\prime}$. Il $\mathrm{cn}$ sera de même de la courbe de Galton, la tangente $\mathrm{BC}^{\prime \prime}$ ou $\mathrm{BC}^{\prime \prime \prime}$ pouvant être voisine de la tangente Gumber, ou située plus ou moins en dessus ou en dessous.

La courbe de Gauss, dans la plupart des cas, donne la valeur de la variable correspondant à une probabilité 1/T très petite, par défaut. Il est vraisemblable qu'à partir d'une certaine probabilité 1/T, les courbes de Galton el de Gumber doivent donner cette valeur par excès, étant domné que la courbe réelle doit s'incurver plus ou moins et tendre à devenir asymptotique à une droite $x_{m}=\mathrm{M}_{0}$, comme l'admet a priori la formule de HORTON.

On se trouve ainsi, quand on veut extrapoler une courbe de fréquence expérimentale, bien au= delà des iimites de probabilités atteintes par l'observation (par exemple dans la prévision des débits extrêmes des cours d'eau), devant une double indécision : quelle formule thérique choisir pour extrapoler la courbe expérimentale? La meilleure courbe étant supposée connue et choisie, à partir de quelle probabilité tres petite $\left(1 /{ }^{\top} \mathrm{T}\right)$ devrons-nous admettre, suivant la loi unique de hasard, le débit impossible? En d'autres termes, quelle est la probabilité du débit que nous considérerons comme "débit maximum du cours d'eau $»$ ?

Toutes les vérités, toutes les prévisions ne sont pas accessibles à l'entendement humain, et il est des problèmes que, pas plus que le déterminisme, le probabilisme ne peut résoudre, et nous nous trouvons ici, précisément, devant un de ces problèmes : le débit maximum d'un cours d'eau est un fait trop particulier... « nos lois et les prévisions que nous fondons sur elles se limitent à des moyennes, ou encore, si l'on veut, à des «Fourchettes».

ETUDE DES « COMPOSANTES »
D'UNE DISTRIBUTION STATISTIOUE

Dans un ordre d'idées analogue, nous estimons que c'est souvent une utopie de vouloir interpréter une distribution statistique au moyen d'une formule théorique unique. Il y a des distributions statistiques qui comportent plus d'une valeur dominante, des courbes de probabilité à plus d'un maximum. Elles sont fréquentes en biologie animale et végétale. Il en existe en météorologie, en climatologie, en hydrologie. C'est ce qu'on appelle le dimorphisme, le polymorphisme. La décomposition d'une courbe de probabilité en deux on plusieurs composantes élémentaires, différenciant les variations de la variable statistique, constitue un problème qui mérite de retenir l'attention des hydrologues.

Pearson et d'autres ont, je crois, étudié la question du point de vue purement mathématique. A défaut d'une telle solution, généralement inabordable anec les données expérimentales en nombre trop réduit que nous possédons en hydrologie fluviale, on peut souvent, par des consi- 
dérations d'ordre météorologique ou hydrologique, d'ordre extra-mathématique par conséquent, envisager et étudier à part deux statistiques, au lieu d'une seule. Tel est le cas, par exemple, pour la distribution statistique de la température journalière : on peut étudier à part la distribution estivale (les six mois les plus chauds) et la distribution hivernale (les six mois les plus froids) : elles ont chacune leur valeur dominante et ces deux valeurs dominantes apparaissent plus ou moins dans la courbe de probabilité générale, qui comporte alors deux maxima distincts. De même, pour les cours d'eau alpestres, serait-il plus indiqué de considérer à part les distribu- tions statistiques du débit hivernal et du débit estival. Aussi bien, quand on se propose de prévoir le débit d'un cours d'eau, uniquement par des considérations de probabilité, on est amené incontestablement à établir les 12 courbes mensuelles individuelles.

La courbe de probabilité d'une variable parfaitement sinusoüdale (cas purement théorique d'ailleurs), nous devrions dire plus exactement sa courbe de fréquence, présente deux maxima. II est vrai qu'une telle variable, en liaison fonctionnelle parfaite avec le facteur temps, cesse alor's d'être une variable statistique plus ou moins conditionnée par le hasard.

\section{III. - L'ÉTUDE DES CORRÉLATIONS ET COVARIATIONS}

Avec les « corrélations », nous abordons un autre ordre de problèmes où la discipline mathématique a également un ròle important à jouer dans les investigations et les prévisions fluviales. Comme nous le verrons d'ailleurs, la notion de «corrélation » complète la notion de «probabilité $>$ en ce sens qu'une formule de corrélation n'est qu'une formale de probabilité, localisée dans le temps ou dans l'espace, ou particularisée, compte tenu des facteurs locaux, géographiques, physiques, climatiques qui interviennent dans le conditionnement du phénomène considéré.

Soit un phénomène concrétisé par une variable statistique $(y)$. Au lieu d'étudier ses variations dans le temps, facteur de durée, c'est-ì-dire en somme en fonction de sa probabilité : $y=f(p)$, indépendamment des facteurs qui le conditionnent, nous étudions ses variations en fonction d'un ou plusieur's des facteur's $(x, z, u \ldots)$ dont il dépend : $y=f(x) \ldots y=f(x, z, u \ldots)$ et plus précisément - étant donné que de telles relations ne sont que des relations empiriques, contenant un terme aléatoire (correspondant à tous les facteurs non considérés individucllement) -..en fonction d'un certain nombre de facteurs, et de la probabilité :

$$
y=\mathrm{F}(x, p) \quad y=\mathrm{F}(x, z, u \ldots p)
$$

si les écarts entre les valeurs observées de $y$ of les valeur's calculées $f(x) \ldots f(x, z, n \ldots)$ suivent la loi des erreurs fortuites, ces formules s'écrivent :

$$
y=f(x)+\frac{z}{\mathrm{~K}} \quad y=f(x, z, u)+\frac{z}{\mathrm{~K}}
$$

La corrélation entre $y$ et $x$, entre $y$ et $x, z, n$. sera d'autant plus forte que le coefficient $K$ sera plus grand, ct d'autant plus intéressante à considérer, en particulier, que $K$ sera plus grand que le cofficient $K_{0}$ de la distribution statistique de $y$ (supposée gaussienne pour fixer les idées) :

$$
y=y_{0}+\frac{z}{\mathrm{~K}_{0}} .
$$

En d'autres termes, à la valeur moyenne $y_{0}$, se trouve substituce, grâce a la corrélation entre $y$ et $x$, entre $y$ et $x, z, u$, une valeur moyenne $f(x), f(x, z, u)$, particularisée suivant chaque valeur de $x$, de $x, z, u$ el d'autant moins aléatoire que $K$ sera plus grand par rapport a $K_{0}$.

Précisons ce que nous venons de dire en considérant plus particulièrement deux variables $y$ et $x$ seulement.

Soient deux phénomènes, simullanés ou décalés l'un par rapport à l'autre dans le temps, se manifestant soit en un même lieu, soit en des lieux différents. On se propose d'étudier s’il existe entre ces deux phénomenes une liaison quelconque, plus ou moins étroite, résultant soit du fait que T'un d'eux est effectivement conditionné par' l'autre plus ou moins, soit du fait qu'ils sont conditionnés l'un et l'autre par un troisième phénomène, ou un ensemble d'autres phénomènes agissant simultanément et parallèlement sur l'un et l'autre, d'une manière prépondérante.

Désignons par $x$ et $y$ les variables statistiques concrétisant ces deux phénomènes. On connaît, par l'observation, $n$ paires de valeur's particulières de $x$ et $y$, soit $x_{1} x_{2} \ldots x_{\mu} \ldots y_{1} y_{2} \ldots y_{n}$.

On peut représenter ces résultats par $n$ points du plan. L'examen d'un tel ensemble de points, formant une «stellaire », un nuage de points conduit à l'une des constatations suivantes :

$1^{\circ}$ Les points sont exactement alignés sur une courbe à laquelle on peut assigner une expression 
algébrique, ou que l'on peut représenter purement et simplement par un tracé empirique ou traduire par un barème.

Nous dirons que les variables $x$ et $y$ sont liées par une relation; qu'il $y$ a entre elles une liaison fonctionnelle traduisant généralement une loi physique rigoureuse.

$$
y=f(x)
$$

2" Les points se trouvent dispersés, dune manière quelconque, dans tout le plan sans présenter la moindre tendance à l'agrégation. Nous dirons que les deux phénomènes $x$ et $y$ ne présentent aucune liaison de quelque nature que ce soit, que la connaissance de $x$ n'apporte rien à la connaissance (et éventuellement à la prévision) de $y$.'L'étude de la variable $y$ se réduit à celle de sa distribution statistique, indépendamment de la variable $x$ : ce que nous traduisons, par exemple, par la formule schématique :

$$
y=y_{0} \pm e
$$

$y_{0}$ étant la valeur moyenne, éventuellement la plus probable et $\frac{\Xi|e|}{n}$ l'écart moyen de probabilité.

$3^{\circ}$ Les points, tout en présentant une certaine dispersion, sont plus ou moins groupés au voisinage d'une courbe, qu'on peut tracer graphiquement et à laquelle on peut assigner, parfois, une expression algébrique.

Nous dirons que les variables $x$ et $y$ sont liées par une « corrélation », c'est-à-dire par une relation plus ou moins lâche, plus on moins aléatoire, impliquant soit une liaison fonctionnelle effective plus ou moins troublée par des erreurs accidentelles d'observation, ou par d'autres facteurs agissant conjointement, soit une simple covariation. La dépendance entre les phénomènes

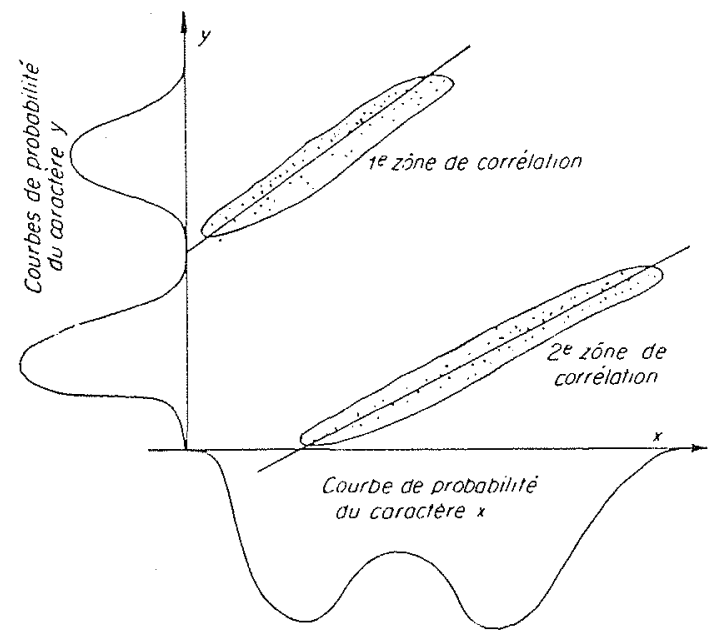

[i. 7 . naturels est évidemment trís complexe, en ce sens que 2, 3, $4 \ldots$ phénomènes réagissent les uns sur les autres, sans que nous puissions les discréminer.

Nous schématisons cette corrélation par la formule :

$$
y=f(x) \pm \varepsilon
$$

$\frac{\Sigma|\varepsilon|}{n}$ sera ce que nous appelons l'écart moyen de corrélation.

Eventuellement, le graphique mettra en évidence deux (ou plusieurs) zones de dispersion, de corrélations distinctes. Nous dirons que la corrélation est dimorphe (ou polymorphe) et nous étudierons séparément les deux corrélations, exprimées par deux formules différentes, ou simplement interprétées par deux courbes différentes. Ce dédoublement, ce dimorphisme montre que le phénomène $(y)$ se dédouble en deux phénomènes distincts, conditionnés différemment par l'ensemble des autres facteurs, ou peut être étudié comme tel. En biologie, cela prouvera que l'espèce étudiée, dont on considère l'un des caractères $y$ comparativement à un autre caractère $x$, comprend effectivement deux espèces, deux variétés plus ou moins distinctes.

On notera que, dans ce cas, il y aura également dimorphisme dans les courbes de probabilité des caractères $y$ et $x$, dimorphisme qui, s'il est asse $z$ accentué, fera apparaitre, dans ces courbes, deux maxima.

Le polymorphisme peut résulter, également, de l'intervention d'un troisième phénomine $(z)$ que

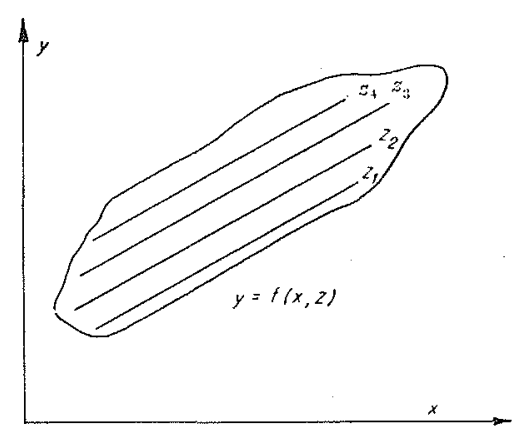

FIG. 8.

le graphique permet de déceler. A chaçue vileur de $z$, à chaque groupe de valeurs de $z$ plus ou moins groupées, correspondront des corrélations différentes entre $y$ et $x: y=f\left(x, z_{1}\right), y=f\left(x, z_{2}\right)$ ete... A la limite on sera conduit a une formule générale $y=f(x, z)$ ou $f(x, z) \pm$ a exprimant une corrélation complexe entre $y, x$ et $z$.

En résumé, la comparaison statistique des valeurs $x_{1} x_{2} \ldots x_{n} \ldots y_{1} y_{2} y_{n}$ fait apparaittre entre 
les deux phénomènes soit une liaison fonctionnelle, soit une liaison mi-fonctionnelle, mi-aléatoire (corrélation ou covariation), soit une liaison nulle, ce que nous schématisons comme il suit :
$y=f(x)$
$y=f(x)$
$y=f(x)$
$y=f(x) \pm z y=f(x, p)$
$y=f(x)+\frac{z}{\mathrm{~K}}$
(3)
$y=y_{0} \doteq e \quad y=f(p)$
$y=y_{0}+\frac{z}{\mathrm{~K}_{0}}$

Disons encore que la formule (3) définit le champ de variation de la variable $y$ considérée isolément, indépendamment de tout autre phénomène ou conditions particulières de temps et de lieu; que la formule (2) définit, pour chaque valeur particulière de $x$, le champ de variation de la variable $y$, d'autant plus réduit par rapport au précédent que le phénomène $x$ interviendra davantage dans le conditionnoment du phénomène $y$, et que la formule (1) correspond au cas-limite où la corrélation fait place à une relation proprement dite rigoureuse.

Ce dernier cas est plus théorique que réel. C'est celui dụ déterminisme absolu, qui, à supposer qu'il existe, nous est absolument inaccessible. "Toutes nos connaissances et les prévisions que nous fondons sur elles ne conduisent jamais à la certitude, mais à des espérances, c'est.à-dire à des probabilités ( $3^{\circ}$ cas), éventuellement améliorées $\left(2^{\circ}\right.$ cas) par des corrélations. La physique ne peut pas prédire les événements futurs avec exactitude, mais seulement les événements possibles et leurs probabilités relatives : la nature ne nous présente pas de lois causales, mais de simples lois de probabilités.

Comme on le voit, les "formules de corrélation » ne sont que des «formales de probabilités $\gg$ particularisées. Et nous ajoutons que toutes les «formules hydrologiques 》 (ou climatiques), qualifiées parfois irrévérentieusement de "recettes hydrologiques $»$, sont des formules de «corrélation » et peuvent et doivent êtie établies comme telles.

Nous distinguons deux sortes de correlations : celles résultant effectivement d'une interdépendance des phénomènes $x$ et $y$, l'un des phénomènes étant effectivement conditionné par l'autre, d'une manière prépondérante, et celles n'impliquant aucune liaison fonctionnelle, mais exprimant seulement une simple covariation de deux phénomènes qui n'ont entre eux d'autre lien que leur déroulement historique.

En d'autres termes, la corrólation entre $\mathrm{A}$ et $\mathrm{B}$ peut être directe: A est l'effet résultant de la cause $B$, ou indirecte: $A$ et $B$ sont les etfets si- multanés ou parallèles d'un troisième phénomène ou ensemble d'autres phénomènes C. Cette distinction n'est d'ailleurs pas toujours très nette.

Exemples. - La corrélation est directe entre les débits et les précipitations d'un bassin.

Il y a une corrélation indirecte, c'est-à-dire une simple covariation entre les débits de deux cours d'eau voisins, soumis aux mèmes influences climatiques générales.

Il y a entre le débit glaciaire et la température une corrélation qu'on peut qualifier soit de directe, soit d'indirecte.

Comme on le voit [nous devons préciser ce point parce que les termes de «corrélation», de méthode de corrélation $»$, de coefficient de corrélation » sont employés, suivant les cas, dans un sens plus ou moins précis et limitatif], nous employons le mot « corrélation » dans un sens à la fois général et élémentaire, indépendamment de toute considération inspirée » par l'ambition de conclure quoi que ce soit touchant la dépendance physique de deux phénomènes : ceci constitue un genre d'études plus abstraites reposant sur une théorie moins élémentaire des corrélations qui dépasse le cadre du présent exposé.

Ainsi envisagée par nous, la notion des corrélations s'étend également :

$1^{\circ}$ A u cas de plus de deux variables étudiées conjointement.

$2^{\circ}$ Au cas où la deuxième variable, ou l'une des variables en fonction desquelles on étudie le phénomène $y$ est le temps, considéré soit comme facteur saisonnier, soit comme facteur périodique général.

$$
y=f(t) \quad y=f(x, z, a, t \ldots)
$$

Nous qualifions une telle corrélation de $p e-$ riodique ou saisonnière, les autres étant dites physiques, hydrométéorologiques, ou géographiques. On l'appelle plus généralement « Périodicité $\gg$.

Une loi périodique quelconque, nécessairement approximative, done aleatoire, par exemple celle reliant la température à l'époque de l'année, du fait du cycle solaire annuel, d'où déconle la notion d'année moyenne thermique, n'est autre qu'une corrélation entre la température et le temps, facteur saisonnier. La notion d'année moyenne, en ce qui concerne tel ou tel élément climatique ou hydrologique, est d'autant plus réelle que la corrélation entre cet élément et sa situation dans l'année est plus grande.

Comment apprécier la valeur d'une corrélation, et si on fonde une prévision sur elle, la prévision et l'utilité de cette prévision? 
Tout simplement en comparant les distributions statistiques des écarts de corrélation $\varepsilon_{1} \varepsilon_{2} \ldots \varepsilon_{n}$ et des écarts de probabilité $e_{1} e_{2} \ldots e_{n}$. La corrélation sera d'autant plus forte que les $\varepsilon$ seront plus petits que les $e$. Elle sera done qualifiée, par exemple, par le coefficient :

$$
r=1-\frac{\mathrm{C}}{\mathrm{P}}=1-\frac{\mathrm{\Sigma}|\varepsilon|}{\Sigma|e|}
$$

que nous appelons « degré de la corrélation» ou rendement de la prévision, si prévision il y a. Au lieu des écarts moyens, on pourra considérer tout aussi bien les écarts-types ou probables.

$\mathrm{Si}$ les écarts $\varepsilon$ et $e$ suivent la loi des erreurs fortuites et si on a, par conséquent :

$$
y=y_{0}+\frac{z}{\mathrm{~K}_{0}} \quad y=f(x)+\frac{z}{\mathrm{~K}_{1}}
$$

le coefficient $r$ sera égal à :

$$
\left(1-\frac{K_{0}}{K_{1}}\right)
$$

L'étude corrélative de deux variables $x$ et $y$, la corrélation éventuelle entre elles étant supposée linéaire, repose essentiellement sur la méthode des moindres carrés, dont nous rappelons le principe :

La relation entre une variable $y$ et d'autres variables $x, z \ldots$ étant supposée de la forme : $y=\alpha+\beta x+\gamma z$ (en ne considérant que trois variables pour fixer les idées), on possède $n$ observations des variables $y_{1} x_{1} z_{1} \ldots y_{n} x_{n} z_{n}$. On multiplie chaque égalité partielle par le coefficient de $\alpha, \beta, \gamma$, et on ajoute. On obtient les valeurs $\alpha \beta \gamma$, donnant l'écart probable minimum, en résolvant les trois équations ainsi obtenues, qui seront :

$$
\left\{\begin{array}{l}
\Sigma y=n \alpha+\beta \Sigma x+\gamma \Sigma z \\
\Sigma y z=\alpha \Sigma x+\beta \Sigma x^{2}+\gamma \Sigma z x \\
\Sigma y z=\alpha \Sigma z+\beta \Sigma x z+\gamma \Sigma z^{2}
\end{array}\right.
$$

Considérons le cas de deux variables entre lesquelles il existe une corrélation linéaire :

\section{On a :}

$\mathrm{Y}=a+b \mathrm{X}$ ou $\mathrm{Y}-\mathrm{Y}_{0}=\lambda\left(\mathrm{X}-\mathrm{X}_{0}\right)$ ou $y=\lambda x$ $\mathrm{X}_{0}$ et $\mathrm{Y}_{0}$ étant les valeurs moyennes des variables $\mathrm{X}$ et $\mathrm{Y}$.

Si on considère $y$ comme fonction de $x$, la valeur de $\lambda$ donnant l'écart probable minimum sera, comme indiqué ci-dessus :

$$
\lambda_{1}=\frac{\Sigma y x}{\Sigma x^{2}}
$$

Si l'on considère $x$ comme fonction de $y$, ce sera :

$$
\lambda_{2}=\frac{\Sigma y^{2}}{\Sigma x y}
$$

La corrćlation sera d'autant plus forte que ces deux valeurs de $\lambda$ seront plus voisines, que les deux droites $y=\lambda_{1} x$ et $y=\lambda_{2} x$ seront plus rapprochées. Si $\lambda_{1}=\lambda_{2}$, les deux droites seront confondues, et il $y$ aura entre $y$ et $x$ une liaison fonctionnelle parfaite, ici linéaire.

On qualifie la corrélation par le rapport :

$$
\sqrt{\frac{\lambda_{1}}{\lambda_{2}}}=\frac{\Sigma y x}{\sqrt{\Sigma x^{2} \Sigma y^{2}}}=\mathrm{R} \text {. }
$$

On a d'ailleurs, dans ce cas, entre A et le coefficient $r$ précédemment défini, la relation théorique :

$$
\mathrm{R}^{2}=1-(1-r)^{2}
$$

Ce calcul constitue le principe, ce coefficient $R$ la pierre angulaire de la méthode élémentaire de corrélation, telle que l'a exposée, par exemple, DE Montessus de Ballore. (La Méthode de Corrélation, collection Gauthier-Villars, 1932.) Comme l'a fait remarquer DEdEBANT : «Appuyé sur de simples considérations de moindres carrés, dépouillé de la signification statistique qu'il tient du calcul des probabilités, ce coefficient n'est plus qu'un indice mesurant le degré de ressemblance entre deux courbes expérimentales, sans qu'on ait le droit de conclure quoi que ce soit touchant la dépendance physique de deux phénomènes dont les mesures ont donné lieu à ces courbes... un simple truc pour juger dans quelle mesure deux courbes se ressemblent. Il mesure la covariation de deux phénomènes mais n'apprend rien de leur dépendance. »

Malgré ces réserves, la considération de ce coefficient se légitime parfaitement, dans de nombreuses applications, tout au moins à titre de sondage préliminaire, notamment quand on se préoccupe seulement de la covariation de deux phénomènes et non de leur dépendance.

Il serait infiniment plus logique de l'appeler «coefficient de covariation » pour éviter toute équivoque. Toutefois, nous lui préférons le coefficient 2 , plus général, et s'appliquant à toute corrélation, quelle qu'elle soit, linéaire ou autre, simple ou complexe.

Au lieu de la forme :

$$
\mathrm{Y}-\mathrm{Y}_{0}=\lambda\left(\mathrm{X}-\mathrm{X}_{0}\right),
$$


il est souvent préférable de considérer la forme :

$$
\frac{\mathrm{Y}}{\mathrm{Y}_{0}}-1=\mu\left(\frac{\mathrm{X}}{\mathrm{X}_{0}}-1\right)
$$

A $\lambda=1$ correspond une corrélation exprimant une différence constante des variables. (Exemple : Corrélation entre les températures de deux localités.)

A $\mu=1$ correspond une corrélation exprimant la constance du rapport des variables. (Exemple: Corrélation entre les précipitations de deux localités.)

Quant au coefficient $\lambda$ (ou u.), sa valeur numérique résulte de l'application de la méthode des moindres carrés, si la corrélation est utilisée pour calculer $y$ en fonction de $x$, on prendra :

$$
\lambda_{1}=\frac{\Sigma x y}{\Sigma x^{2}}
$$

Dans le cas contraire :

$$
\lambda_{2}=\frac{\Sigma y^{2}}{\Sigma x y}
$$

On peut également, de la considération des deux courbes de probabilité des variables $y$ et $z$, déduire la corrélation qui existe entre elles, indépendamment du sens dans lequel la corrélation est utilisée. Soit les deux distributions supposées gaussiennes :

$$
z=h_{1}\left(\mathrm{X}-\mathrm{X}_{0}\right) \quad z=h_{2}\left(\mathrm{Y}-\mathrm{Y}_{0}\right)
$$

Eliminant $z$ entre ces deux équations, on obtient :

$$
\mathrm{Y}-\mathrm{Y}_{0}=\frac{h_{1}}{h_{2}}\left(\mathrm{X}-\mathrm{X}_{0}\right)
$$

$\frac{h_{1}}{h_{2}}$ est égal soit au rapport des écarts moyens, soit au rapport des écarts probables; c'est-à-dire qu'on a :

$\lambda^{\prime}=\frac{h_{1}}{h_{2}}=\frac{\Sigma\left|\mathrm{Y}-\mathrm{Y}_{0}\right|}{\Sigma\left|\mathrm{X}-\mathrm{X}_{0}\right|} \quad$ ou $\quad \lambda^{\prime \prime}=\sqrt{\frac{\Sigma\left(\mathrm{Y}-\mathrm{Y}_{0}\right)^{2}}{\Sigma\left(\mathrm{X}-\mathrm{X}_{0}\right)^{2}}}$

Si la corrélation est forte - et c'est seulement dans ce cas qu'il peut ètre utile de la considérer, en vue notamment d'une prévision, les valeurs $\lambda_{1} \lambda_{2} \lambda^{\prime}$ et $\lambda^{\prime \prime}$ sont très voisines l'une de l'autre.

L'étude d'une corrélation plus complexe entre $\mathrm{Y}$ et $\mathrm{X}$, c'est-à-dire non linéaire, peut souvent, par un changement de variables, ètre ramenée à l'étude d'une corrélation linéaire.

Ainsi en est-il d'une corrélation parabolique:

$$
-\frac{\mathrm{Y}}{\mathrm{Y}_{0}}=\left(\frac{\mathrm{X}}{\mathrm{X}_{0}}\right)^{\mathrm{K}}
$$

ou exponentielle :

$$
\mathrm{Y}-\mathrm{Y}_{0}=e^{\mathrm{K}\left(\mathrm{X}-\mathrm{x}_{0}\right)},
$$

qui peuvent être considérés comme une corrélation entre $\log \mathrm{Y}$ et $\log \mathrm{X}$ ou entre $\log \mathrm{Y}$ et $\mathrm{X}$.

De même une corrélation $\mathrm{Y}=a \mathrm{X}+b \mathrm{X}^{2}$ pourra être mise sous la forme:

$$
\left(\frac{\mathrm{Y}}{\mathrm{X}}\right)=a+b \mathrm{X}
$$

On se contente généralement d'exprimer une corrélation linéaire par la formule simple :

$$
\mathrm{Y}-\mathrm{Y}_{0}=\lambda\left(\mathrm{X}-\mathrm{X}_{0}\right) \text {. }
$$

Si le nombre des observations est suffisant, on pourra, par la considération des écarts, compléter la formule, en $y$ introduisant la variable «Probabilité »:

$$
\mathrm{Y}-\mathrm{Y}_{0}=\lambda\left(\mathrm{X}-\mathrm{X}_{0}\right)+f(p)
$$

soit :

$$
\mathrm{Y}-\mathrm{Y}_{0}=\lambda\left(\mathrm{X}-\mathrm{X}_{0}\right)+\frac{\mathrm{Z}}{\mathrm{K}}
$$

si la distribution est gaussienne. Cela revient à graduer la zone de dispersion, de corrélation par des courbes d'égales probabilités, en d'autres termes à tracer l'abaque $\mathrm{Y}=f(x, p)$, soit simplement graphique, soit interprétée algébriquement.

CORRÉlATIONS ENTRE PLUS DE DEUX VARIABLES

Il ne saurait y avoir de règle générale pour exprimer empiriquement, c'est-à-dire compte tenu des seules statistiques, une variation $y$ en fonction de deux ou plus de deux autres variables : $y=f(x, z, u \ldots)$. Ciest là un domaine d'investigations où l'ingéniosité et le flair des chercheurs peuvent s'exercer, et de fait se sont exercés librement. Le problème ne comporte d'ailleurs pas une solution unique. La considération du rapport $r$ permet, entre deux formules différentes, de juger quelle est la meilleure: ce sera celle dans laquelle le facteur $K$ du terme aléatoire $z / K$ par exemple sera le plus grand. Dans la recherche de telles corrélations, il sera d'ailleurs nécessaire, le plus souvent, de faire intervenir des considérations « extra-mathématiques » inspirées par la nature des faits observés, soit hydrologiques, soit géographiques.

"Dans une expérience organisée au Laboratoire, a écrit G. Dedebant (Statistique et Corrélations, la Météorologie, mai 1934), il est possible de ne conserver qu'une variable indépendante, mais dans les sciences de la nature, où l'expéri- 
mentation consiste à attendre, pour les observer, des événements qu'on ne provoque pas, il y aura toujours plus d'une variable indépendante.

Peut-on cependant tirer parti d'observations laites dans ces conditions?

Oui, si nous voulons bien élargir la notion de dépendance au-delà de la liaison fonctionnelle. Ainsi la hauteur du baromètre en un lieu ne détermine pas, à coup sûr, le temps qu'il va faire, mais l'on sait pourtant qu'il y a plus de chances de pluie et de tempête par basses pressions que par hautes pressions.

Cette dépendance, assez lâche, à vrai dire, entre le temps et la pression barométrique en un lieu, a été mise depuis longtemps à profit par les constructeurs, qui graduent les cadrans des baromètres anéroïdes selon le caractère du temps : grand vent, pluie, variable, sec, etc...

Pourquoi les hommes de science seraient-ils les seuls à ne pas vouloir envisager ce genre de dépendances, surtout dans les sciences complexes dont la météorologie est le type, où, vu le nombre des variables qui conditionnent la marche du moindre des phénomènes, et qu'on ne peut espérer connaître toutes, la plupart des relations perceptibles offrent un caractère aléatoire.

On dit que ce qui distingue l'esprit géométrique de l'esprit de finesse à la manière de Pascar, c'est que le premier ne perçoit que les liaisons fonctionnelles, tandis que le second voit et pèse les dépendances plus lâches et sait en tirer parti. L'esprit de finesse doit-il ètre par principe refusé aux géomètres? Non, et nulle part qu'en calcul de probabilités ils n'ont mieux montré qu'ils en étaient pourvus. »

Ainsi donc, de proche en proche, par l'étude des probabilités et des corrélations de plus en plus particularisées rendue possible par la multiplication de nos observations, nous nous effor- cerons de connaitre plus à fond la nature, le conditionnement de tel ou tel phénomène, en vue, éventuellement, d'en prévoir les manifeslations ultérieures, pour les utiliser, ou pour nous en défendre.

"Parviendrons-nous un jour à une connaissance de tous les facteurs météorologiques ( $A n-$ ders Angtröm) et de leurs relations mutuelles suffisante. pour nous permettre, par exemple, d'après notre connaissance de $x, z, u$..., d'obtenir une valeur exacte de $y$ et de ses variations futures? Nous pouvons espérer une réduction de notre marge d'erreurs, par l'introduction de nouveaux facteurs, dans notre système d'observations; cependant, le nombre des facteurs qui échappent à nos mesures demeurera nécessairement si grand que nos prévisions se réaliseront toujours avec un certain degré de probabilité, non de certitude.

Dans le même ordre d'idées, Porncaré a écrit : * Ainsi, grâce à la généralisation, chaque fait observé nous en fait prévoir un grand nombre; seulement nous ne devons pas oublier que le premier seul est certain, que tous les autres ne sont que probables. Si solidement assise que puisse nous paraitre une prévision, nous ne sommes jamais sûrs absolument que l'expérience ne la démentira pas, si nous entreprenons de la vérifier. Mais la probabilité est souvent assez grande pour que nous puissions nous en contenter. Mieux vaut prévoir sans certitude que de ne pas prévoir du tout.

De ce que nous pouvons atteindre, il rant tirer le meilleur parti : il faut que chaque expérience nous permette le plus grand nombre possible de prévisions, et avec le plus haut degré de probabilité qu'il se pourra. Le problème est pour ainsi dire d'augmenter le rendement de la machine scientifique.»

\section{IV. - DES PRÉVISIONS}

De l'exposé que nous venons de faire, nous dégagerons, du point de vue des Prévisions, notamment fluviales, les quelques conclusions générales suivantes :

$1^{\circ}$ Toute prévision est fondée sur une courbe de probabilité plus ou moins particularisée, compte tenu de certaines corrélations d'ordre hydrométéorologique, géographique, périorlique : tout phénomène se passe en un lieu, à une ipoque donnée, et en ce lieu, à cette époque, y est occasionné par un certain nombre de phénomènes.
Une telle courbe de probabilité peut se schématiser par une formule :

$$
y=\mathbf{F}(x, z, u, t \ldots p)
$$

ou $y=f(x, z, u, t)+\varphi(p)$ avec $\varphi(p)=\frac{z}{\mathbf{K}}$ si les écarts de corrélation suivent la loi de Gauss.

2" Si l'on entend le mot «prévision » dans son sens le plus général, comprenant les interpolations de toute nature (dans le temps comme dans l'espace), soit « determination de certuins 
faits ou phénomènes passés, actuels ou futurs, non directement observés ou observables, en fonction de certains autres faits ou phénomènes permanents ou variables, observés ou observables directement », on peut dire que toute formule hydrologique de ce genre est susceptible de servir à des prévisions.

$3^{\circ}$ S'il s'agit de prévisions proprement dites, il faut naturellement que les facteurs $x, z, u, t$ interviennent dans la formule, ou du moins les plus importants, puissent être connus antérienrement au phénomène $y$.

Cette condition - si l'on ne tient pas compte des prévisions d'ordre météorologique, possibles éventuellement, concernant la température, les précipitations futures - limite les prévisions concernant les débits futurs, à plus ou moins longue échéance, au cas où ils sont surtout conditionnés soit par une loi périodique telle que la périodicité annuelle, soit par des facteurs antérieurs, en l'occurrence par les capacités de rétention du bassin, ou en réduit la précision et l'efficacité.

$4^{\circ}$ Pour cette raison, deux sortes de prévisions sont particulièrement utiles à considérer :

a) Celles concernant les débits des bassins où les précipitations présentent un certain caractère périodique : par exemple rareté ou absence de précipitations pendant plusieurs mois consécutifs;

b) Celles concernant les débits printaniers ou estivaux des bassins a forte nivosité hivernale.

$5^{\circ}$ Dans tous les autres cas, les prévisions, beaucoup moins efficaces, reposent sur la considération des courbes de probabilité mensuelles, faisant intervenir la périodicité annuelle, soit :

$$
\begin{gathered}
\mathrm{Q}_{n}=f\left(t_{n}, p\right)\left[t_{n}=\text { le mois de rang } n\right] \\
\text { - soit } \frac{\mathrm{Q}_{n}}{\mathrm{Q}_{n-1}}=f\left(t_{n}, p\right)
\end{gathered}
$$

cette dernière formule tenant compte, dans une certaine mesure, de l'état antérieur du cours d'eau. $\left(Q_{n}\right.$ et $Q_{n-1}=$ débits du mois et du mois antérieur.)

Plus l'écoulement sera périodique saisonnièrement, c'est-à-dire plus il sera conditionné par la température (facteur beaucoup plus périodique que le facteur Précipitations dans nos régions tempérées), c'est-à-dire plus il sera nivoglaciaire, plus de telles prévisions seront utiles à considérer.

L'exemple suivant schématique montrera com- ment, en particularisant de plus en plus la courbe de probabilité, on peut espérer diminuer le champ de variation de la variable « Débit » à prévoir.

On se propose de prévoir le débit d'un mois quelconque ì venir.

1" On ne comnait que la courbe de probabilité générale donnant le débit mensuel en fonction de sa probabilité : $q=q_{0}+z / K$.

En prévoyant que le débit sera égal à $q_{0}$, l'erreur moyenne sera par exemple : $\mathrm{E}_{0}$.

2" L'utilisation de deux courbes distinctes, l'une concernant la saison hivernale, l'autre la saison estivale, $q=q_{0}^{\prime}+z / \mathrm{K}^{\prime}$ et $q=q^{\prime \prime}{ }_{0}+z / \mathrm{K}^{\prime \prime}$ réduira cette erreur de $\mathrm{E}_{0} \dot{a} \mathrm{E}_{1}$.

$3^{\circ}$ En considérant les 12 courbes mensuelles de probabilité, soit l'abaque $q=f(t, p)$ - ce qui revient à tirer parti de la périodicité annuelle, l'erreur, encore plus faible, sera $\mathrm{E}_{2}$.

$4^{\circ}$ On possède, pour chaque mois, non pas une seule courbe de probabilité, mais deux, l'une correspondant au cas où le débit du mois antérieur a été déficitaire ( $<$ à la moyenne), et l'atttre au cas où le débit a été excédentaire ( $>$ à la moyenne). En utilisant, suivant le cas, l'une ou l'autre de ces deux courbes, on peut escompter une erreur moyenne plus faible encore, soit $\mathrm{E}_{3}$. On a, ce faisant, utilisé la corrélation interne entre les débils de deux mois successifs.

$5^{\circ}$ En généralisant, on sera amené à uliliser une formule de corrélation: $Q_{n}=f\left(t_{n} Q_{n-1} p\right)$ exprimant le débit du mois $t_{n}$ en fonction de sa probabilité et du débit du mois antérieur : l'erreur est ramenée à $\mathbf{E}_{4}$.

$6^{\circ} \mathrm{Si}$, en outre, le mois sur lequel porte la prévision a son débit conditionné, en majeure partie, par la fusion des neiges hivernales, en tenant compte de la corrélation nivo-fluviale supposée établie d'après des observations antérieures, l'écart moyen pourra encore être réduit et sera, par exemple, égal à $\mathrm{E}_{5}$.

Au fur et à mesure que l'on utilise plus de corrélations (corrélation périodique annuelle, corrélation fluviale intermensuelle, corrélation nivofluviale), l'écart moyen diminue :

$$
\mathrm{E}_{5}<\mathrm{E}_{1}<\mathrm{E}_{3}<\mathrm{E}_{2}<\mathrm{E}_{1}<\mathrm{E}_{0}
$$

et le rendement $r^{\prime}=1-\mathrm{E} / \mathrm{E}_{0}$, de la prévision, augmente.

Cet exemple schématise dogmatiquement la genése d'une prévision, et son amélioration progressive, au fur et à mesure que la mise en ouvre de plus de corrélations permet de réduire le champ de variation du débit à prévoir. 


\section{V. - CONCLUSIONS}

Mais il est temps de terminer cette trop longue causerie. Pour en tirer un enseignement plus général, extraprofessionnel pour ainsi dire, philosophique même, je la terminerai, comme je l'ai commencée, par une citation de M. PAndé, brièvement commentée. Elle concerne la prévision du débit de crue maximum possible :

« Le maximum à craindre, si cette définition possède un sens, peut rester obscur pour les géographes hydrologues les mieux renseignés. Et le mathématicien viendra peut-être utilement à leur secours, si, ne méprisant pas l'examen des faits réels, possédant, outre l'esprit géométrique, celui de finesse, il a su établir d'après des bases numériques assez nombreuses. et ieconnues bonnes après critique, une formule de fréquence qui intègre dans sa moelle l'influence de ioutes les particularites géophysiques de la région. De la sorte, et Dieu aidant (pourquoi pas?) son algebre intelligente résoudra peut-être jusqu'à un certain point le mystère des déluges.»

Oui, messicurs, vous pouvez faire confiance aux mathématiciens, sinon pour accroître, du moins pour coordonner, rationaliser, nos connaissances hydrologiques, les ériger en lois statistiques et faciliter les prévisions que l'on peut fonder sur ces connaissances. Et ce n'est certes pas devant cet auditoire que j'ai à insister sur ce point.

Mais résolveront-ils un jour «le mystère des déluges », comme le souhaite M. PARDÉ, «en intégrant dans une formule tous les facteurs intervenant dans la genèse des crues?... c'est-àdire, plus généralement, à établir une démarcation entre le domaine des Probables et le domaine des Impossibles?... J'en doute, et ne devrons-nous pas nous contenter, longtemps encore, sinon toujours, de la «loi unique de hasard», mentionnée plus haut, qui esquive le problème plutôt qu'elle ne le résoud?

Le mystère serait-il résolu, que d'autres mystères surgiront sous leurs pas, tant il est vrai que la science est évocatrice de mystères, qu'elle en déćouvre plus qu'elle n'en résoud...

Il faut leur faire confiance, en souhaitant, entre eux et les hydrologues proprement dits, une collaboration plus étroite et plus compréhensive.

Mais il faut les excuser, et leur pardonner, si parfois, séduits par les incantations des sirénes qui peuplent les espaces abstraits, ils prêtent une oreille moins attentive, plus distraite aux chants folkloriques des ondines de nos fleuves et rivières, ainsi que le batelier légendaire du Rhin, dé- lourné de ses préoccupatioins fluviales par la mystérieuse mélopée de la « Loréley » déroulant, du haut d'un rocher abrupt, sous ses regards éblouis, le trésor de sa chevelure blonde.

Indépendamment des réalités que les mathématiques se doivent d'expliquer, sans les déformer, cette science possede des beautés propres...

Ecoutons Edgar QuneT célébrer les noces de l'Algèbre et de la Géométrie sous le signe de Descartes :

« Si l'algèbre m'avait frappé, je fus ébloui par l'application de l'algèbre à la géométrie... L'idée, la possibilité d'exprimer une ligne, une courbe, par des termes algébriques, par une équation, me parut aussi belle que l'Iliade. Quand je vis cette équation fonctionner et se résoudre, pour ainsi dire, toute seule entre mes mains et éclater en une infinité de vérilés, toutes également indubitables, également éternelles, également resplendissantes, je crus avoir en ma possession le talisman qui m'ouvrait la porte de tous les mystères.»

"C'est ainsi que la beauté se déploie en mathématiques comme dans les autres sciences, comme dans les arts, comme dans la vie et comme dans la nature. Parfois comparables à celles de la musique pure, de la grande peinture ou de la poésie, les émotions qu'elle éveille sont le plus souvent d'une nature différente qui ne peut guère se comprendre lorsqu'on n'en a pas ressenti soi-même l'illumination. La beauté des mathématiques ne garantit certes ni leur vérité, ni leur utilité. Mais elle apporte aux uns de pouvoir vivre des heures incomparables, aux autres la certitude que les mathématiques continueront à être utilisées pour le plus grand profit de tous, et la plus grande gloire de l'aventure humaine par des hommes qui n'en espèrent pour cux-mèmes aucun profit matériel. »(F. LE LionvaIs.)

Aussi bien aucune discipline scientifique plus que la discipline mathématique n'exige, quoi qu'on pense, plus d'esprit de finesse, en plus de l'esprit géométrique. Sans l'esprit de finesse des mathématiciens, le calcul des Probabilités, le calcul des Corrélations, arec toutes leurs richesses découvertes ou restant encore à explorer, n'auraient jamais vu le jour...

Si vous en avez le loisir et le goût, je vous recommande de parcourir "les grands courants de la Pensée mathématique $»$ de F. Le Lronnass. Vous y trouverez notamment, sous la signature 
de M. Ulumo, M. de C. à l'E. P., un article intitulé : «La position moderne du débat: Esprit géométrique, Esprit de finesse... »

Je livre à vos méditations sa conclusion :

«Nous pouvons maintenant conelure, et renvoyer dos à dos les sectaires de l'esprit de géométrie mal compris, comme ceux de l'esprit de finesse mal interprété. Explicitant la démarche des esprits éclairés, nous avons découvert la synthèse de cette antithèse périméc.

C'est une erreur et une caricature du cartésianisme que de croire que la vérité est atteinte infailliblement par la vertu rigide de la déduction : proposer un mécanisme d'interaction peu probable, e'est pour l'intelligence véritable se tromper autant que proposer un mécanisme absurde : plus généralement, se satisfaire, s'arrêter lorsque la déduction a atteint un mécanisme particulier, croire par un seul coup de filet avoir épuisé le réel, c'est la faute la plus grave, le péché d'orgueil, que la vraie science ne pardonne pas.

G'est une er'eur et une caricature de la finesse pascalienne que d'affirmer, vis-à-vis de la vérité, un scepticisme élégant, de tenir la balance égale entre toutes les thèses. De ces contempteurs de la méthode, qui croient aux prestiges de la forme, et ne croient pas profondément au pouvoir de la raison sur les faits, nous savons bien que les faits se vengent.

La vérité n'est pas une, au bout d'une chaine logique rigide, elle n'est pas indéterminée dans toutes les directions du discours. Elle est une plage délimilée par des axes privilégiés. Reconnaitre ces axes, éclairer cette plage, cest explorer les possibles, et préciser les probables, c'est appliquer aux choses la puissance de clarté et d'ordonnance de l'esprit, en un mot c'est comprendre.»

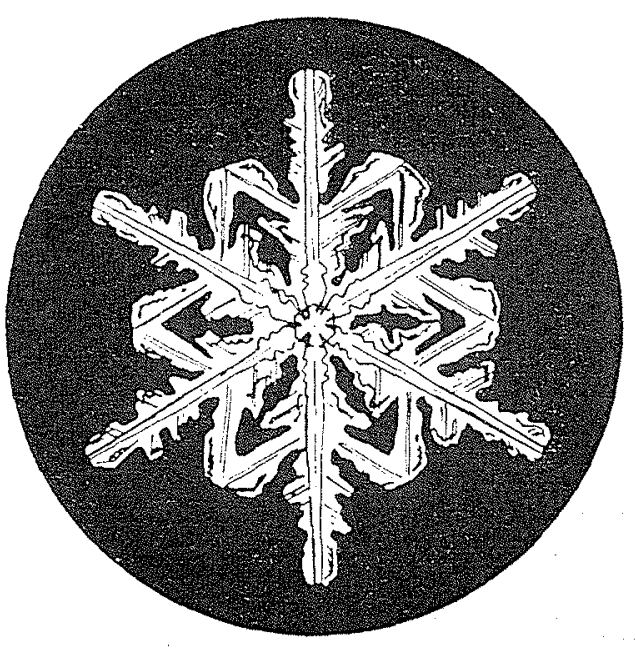

